\title{
20. The relative differential geometry in affine space $\left({ }^{*}\right)$.
}

\author{
By \\ Junkô HiRAKAWA. \\ (Received September 26, 1940.)
}

\section{Introduction.}

In this paper a convex closed curve or a convex closed surface is respectively taken as the unit curve or the unit surface as in euclidean space $\left({ }^{1}\right)$.

In the first chapter a convex closed curve $E(\mathfrak{c})$ is taken as the unit curve and we make correspondence between a point $P$ on a curve and a point $Q$, on the unit curve, so that the tangents at the points $P$ and $Q$ are parallel to each other. Denote by $q$ the affine perpendicular distance to $Q$ on $E$ from the origin $O$. At first I will give a geometrical meaning of the affine perpendicular distance. By using it we define the $r$-affine arc length and try to make a study about the theory of plane curves.

In the second chapter a convex closed surface $E(\mathrm{c})$ is taken as the unit surface and we make correspondence between a point $P$ on a space curve and a point $Q$, on the unit surface, so that the tangent plane at $Q$ is parallel to the affine rectifying plane at $P$. At first the affine perpendicular distance to the osculating plane and to the affine rectifying plane from a point are defined and the affine perpendicular distance $\left({ }^{2}\right)$ of a surface from a point is introduced by the above definition, and consequently a geometrical meaning of this distance is given. By using the affine perpendicular distance of the unit surface we define the $r$ affine arc length and try to make a study about the theory of space curves.

In the third chapter a convex closed surface $E(\mathrm{e})$ is also taken as the unit surface and we make correspondence between a point $P$ on a

(*) This investigation has been made under the financial assistance of the Educational Department.

(1) J. Hirakawa, The Euclidean Relative Differential Geometry; I. Theory of plane curves. Proc. of the Physico Math. Soc. of Japan, 17 (1935) ; II. Theory of space curves. Jap, Journ. of Math., XIII (1936) ; III. Theory of surface-strips. Loc. cit., XIV (1937) ; IV. Theory of surfaces. Loc. cit., XIV (1938).

(2) W. Blaschke, Differential Geometrie, II, p. 110, Affinentfernung. 
surface and a point $Q$ on the unit surface, such that the tangent planes are parallel to each other. At first a relation between the two points $P$ and $Q$ is investigated. In the next place, by using the affine perpendicular distance of $E(\mathfrak{e})$, we define a vector $\xi_{i k}$ by the elementary tensor $g_{i k}$, and try to make a study about the theory of surfaces.

In conclusion I express many thanks to Prof. T. Kubota for his remarks concerning this investigation.

\section{Contents.}

Chapter I. Theory of plane curves.

§1. Affine perpendicular distances and relative affine arc length $\ldots \ldots \ldots 349$

$\S 2 . \quad R$-affine curvatures and fundamental equations. .............. 351

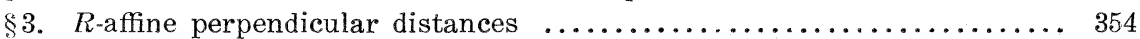

$\S 4$. Indicatrices of tangents and of curvatures. $R$-affine circles $\ldots \ldots \ldots 355$

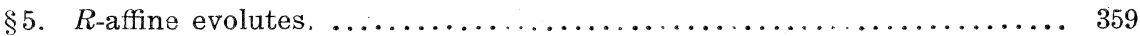

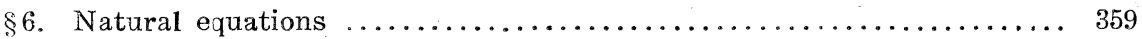

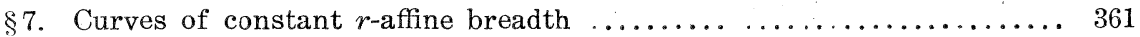

Chapter II. Theory of space curves.

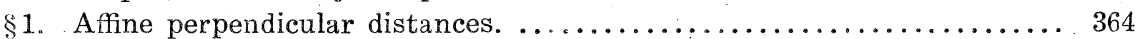

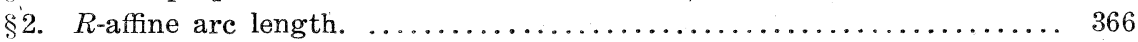

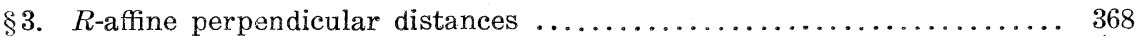

$\S 4$. Indicatrices of tangents, $r$-curvatures and $r$-covariant curvatures. $\ldots 371$

$\$ 5$. A geometrical meaning of the $r$-affine principal normal. ......... 373

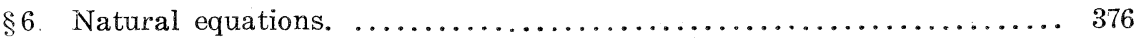

$\$ 7$. Special curves. .......................................... 378

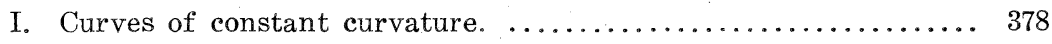

II. Curves of constant torsion. ............................ 378

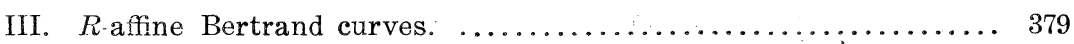

Chapter III. Theory of surfaces. . . . . . . . . . . . . . . . 382

$\S 1$. Relations between two surfaces with their parallel tangent planes. .. 382

$\S 2$. Fundamental quantities and fundamental equations. ........... 383

$\S 3$. $R$-affine lines of curvature and $r$-affine principal curvatures. $\ldots \ldots \ldots 387$

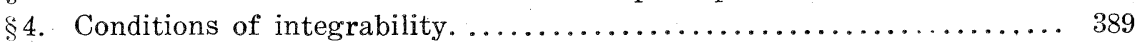

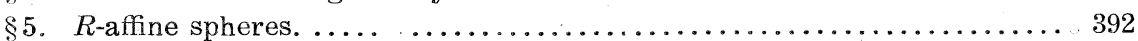

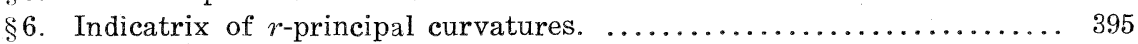

$\$ 7$. Surfaces of constant $r$-affine breadth. ................... 397 


\section{Chapter 1. Theory of plane curves.}

\section{\$1. Affine perpendicular distances and relative affine arc length.}

Take a point $P$ on a plane curve $C$ and denote by $P^{\prime}$ and $P^{\prime \prime}$ two points consecutive to the point $P$. Let us denote the radius vector of $P$ from the origin $O$ by $x(t)$ as a function of a parameter $t$, and denote by $x(t+\Delta t)$ and $\mathfrak{x}(t+\Delta t+\Delta(t+\Delta t))$ respectively the radius vectors $O P^{\prime}$ and $O P^{\prime \prime}$. Take any point $A(\mathfrak{a})$ on this plane, then we have

$$
\text { (1) } \begin{aligned}
\lim _{P^{\prime}, P^{\prime \prime} \rightarrow I^{\circ}} \frac{2 \Delta A P^{\prime} P^{\prime \prime}}{\Delta t} & =\lim _{\Delta t \rightarrow 0} \frac{1}{\Delta t}|\mathfrak{a}-\mathfrak{x}(t+\Delta t), \mathfrak{a}-\mathfrak{x}(t+\Delta t+\Delta(t+\Delta t))| \\
& =\lim _{\Delta t \rightarrow 0} \frac{1}{\Delta t}|\mathfrak{a}-\mathfrak{x}(t+\Delta t),-\dot{x}(t+\Delta t) \Delta(t+\Delta t)-\cdots| \\
& =|\mathfrak{a}-\mathfrak{x}(t),-\dot{x}(t)|=\omega
\end{aligned}
$$

( 2) $\lim _{P^{\prime}, P^{\prime \prime} \rightarrow P} \frac{2 \Delta P P^{\prime} P^{\prime \prime}}{\Delta t^{3}}=\lim _{i \Delta t \rightarrow 0} \frac{1}{\Delta t^{3}}|x(t+\Delta t)-x(t), x(t+\Delta t+j(t+\Delta t))-\mathrm{x}(t)|$

Let us nut

$$
=|\ddot{x}(t), \ddot{\mathrm{x}}(t)| \equiv \sigma^{3} \text {. }
$$

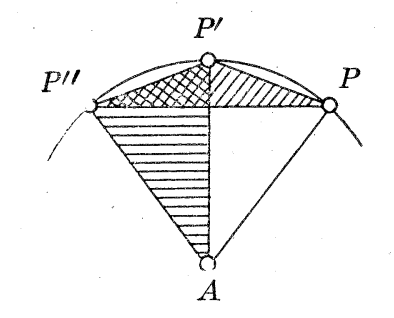

$$
\mathfrak{p}=\frac{\omega}{\sigma}
$$

then we have

$$
\mathfrak{p}=\frac{\omega}{\sigma}=\left|\mathfrak{x}-\mathfrak{a}, \frac{d x}{d \bar{S}}\right|=(\mathfrak{a}-\mathfrak{x}) \xi \cdot\left|\frac{d x}{d \bar{S}}\right|=p\left|\mathfrak{x}^{\prime}\right|=p \bar{\rho}^{\frac{1}{3}},
$$

where $\xi, \bar{S}, p$ and $\bar{\rho}$ denote respectively the unit normal vector, the affine arc length, the perpendicular distance from $A$ to the tangent line at $P$ and the radius of curvature at $P$. Thus we find that $p$ is the same as the "Affinenfternung" which has been introduced by Prof. $W$. Süss $\left({ }^{3}\right)$. Hereafter we call $\mathfrak{p}$ the affine perpendicular distance.

In $\S 3$ we will prove the following

Theorem 1. If the affine perpendicular distance from a fixed point to any point on a given curve $C$ be constant, the curve $C$ is a conic.

Let us make correspondence between a point $P$ on a curve $C$ and a point $Q$ on the unit curve $E($ e) so that the tangent at $P$ and $Q$ are

(3) W. Süss, Zur relativen-Differentialgermetrie I, Jap. Journ. of Math., IV (1927). 
parallel to each other; denote by $q$ the affine perpendicular distance from the origin $O$ to the point $Q$, then we have

$$
\mathfrak{q}=\frac{\omega_{0}}{\sigma_{0}}=(-\mathrm{e} \xi)\left|\frac{d \mathfrak{e}}{d \bar{s}}\right|=q \bar{\rho}_{0}^{\frac{1}{3}},
$$

where $\omega_{0}=|\mathfrak{e}(t), \dot{e}(t)|, \quad \sigma_{0}^{3}=|\dot{\mathfrak{e}}(t), \ddot{e}(t)|$ and $\bar{s}, q$ and $\bar{p}_{0}$ denote respectively the affine arc length, the perpendicular distance and the radius of curvature at $Q$.

The relative affine arc length $d S$ is defined as

$$
d S=\frac{\omega_{0}}{\sigma_{0}} \sigma d t=q d \bar{S}
$$

Now we will prove the following relation:

$$
\frac{d S}{d s}=\frac{d \bar{S}}{d \bar{s}}=\rho^{\frac{2}{3}}
$$

where $\rho$ denotes the radius of $r$-curvature. $\left({ }^{4}\right)$

As the corresponding tangents at $P$ and $Q$ are parallel, we can put

$$
\frac{d r}{d \bar{S}}=\lambda \frac{d \mathfrak{e}}{d \bar{s}}
$$

where $\lambda$ is a positive scalar quantity.

By means of the relations

$$
\left|\frac{d x}{d \bar{S}}\right|=\bar{\rho}(x)^{\frac{1}{3}} \text { and } \quad\left|\frac{d e}{d \bar{s}}\right|=\bar{\rho}(e)^{\frac{1}{3}} \text {, }
$$

we have

$$
\lambda=\left(\frac{\bar{\rho}(x)}{\bar{\rho}(e)}\right)^{\frac{1}{3}}=\rho^{\frac{1}{3}}
$$

then (7) becomes

$$
\frac{d x}{d \bar{S}}=\rho^{\frac{1}{3}} \frac{d e}{d \bar{s}}
$$

By differentiating (1) with respect to $\bar{S}$ and using the relation

$$
\left|\frac{d x}{d \bar{S}}, \frac{d^{2} x}{d \bar{S}^{2}}\right|=1, \text { we obtain }
$$

(4) W. Süss, Zur relativen Differentialgeometrie I, Jap. Journ. of Math., IV. (1927), and J. Hirakawa, loc. cit. (1). 


$$
\frac{d S}{d s}=\frac{d \bar{S}}{d \bar{s}}=\lambda^{2}=\rho^{\frac{2}{3}}
$$

By means of (1), (2) and (9), we obtain the following relation

$$
\frac{\sigma}{\sigma_{0}}=\rho^{\frac{2}{3}}
$$

Taking the $r$-affine arc length $S$ as parameter, we have from (5)

$$
\Sigma=\frac{\Sigma_{0}}{\Omega_{0}}=\frac{1}{\mathfrak{q}}
$$

where $\Sigma_{0}, \Omega_{0}$ and $\Sigma$ denote respectively the values of $\sigma_{0}, \omega_{0}$ and $\sigma$ for the parameter $t=s$.

Therefore we have

$$
\Omega_{0}=\rho^{-\frac{2}{3}}
$$

From (10) by making correspondence to the unit circle we find

$$
\bar{\rho}(x)^{-\frac{2}{3}} d \bar{S}=\bar{\rho}(\mathfrak{e})^{-\frac{2}{3}} d \bar{s}=\text { invariant }=d \theta,\left({ }^{5}\right)
$$

where $\theta$ denotes the angle which the tangent makes with $x$-axis.

\section{$\S 2$. R-affine curvature and fundamental equations.}

Let us put

$$
\frac{d r}{d S}=\ell^{\prime}=\xi_{1}, \text { and } \quad \frac{d^{2} x}{d S^{2}}=\ell^{\prime \prime}=\frac{\xi_{2}}{q^{3}},
$$

then from (12) we have

$$
\left(x^{\prime}, x^{\prime \prime}\right)=\frac{1}{q^{3}}
$$

and

$$
\left(\xi_{1}, \xi_{2}\right)=1 \text {. }
$$

By differentiating (17) with respect to $S$, we have

$$
\left(\xi_{1}, \xi_{2}^{\prime}\right)=0
$$

(5) This relation is taken from (153) and (157) in page 32, W. Blaschke, Diff, Geom. II, (1923). 
then we obtain the fundamental relations:

$$
\xi_{1}^{\prime}=\frac{\xi_{2}}{q^{3}},
$$

and

$$
\xi_{2}^{\prime}+k \xi_{1}=0
$$

where $k$ is called the r-affine curvature.

By means of (17) and (19) $k$ is found from the equation

$$
k=\left(\xi_{2}, \xi_{2}^{\prime}\right)=q^{3}\left(\xi_{2}, \xi_{1}^{\prime \prime}\right) .
$$

From (18) we have

$$
\left(\xi_{1}^{\prime}, \xi_{2}^{\prime}\right)+\left(\xi_{1}, \xi_{2}^{\prime \prime}\right)=0,
$$

and

$$
\left(\xi_{1}^{\prime}, \xi_{2}^{\prime}\right)=\left(q^{3} r^{\prime \prime}, x^{\prime \prime \prime}\right)=\left(\xi_{2}, \xi_{1}^{\prime \prime}\right)
$$

Thus the following relations are obtained :

$$
\left(\xi_{2}, \xi_{1}^{\prime \prime}\right)=\left(\xi_{2}^{\prime \prime}, \xi_{1}\right)
$$

and

$$
k=\left(\xi_{2}, \xi_{2}^{\prime}\right)=q^{3}\left(\xi_{2}, \xi_{1}^{\prime \prime}\right)=\mathfrak{q}^{3}\left(\xi_{2}^{\prime \prime}, \xi_{1}\right) .
$$

In the next place we will seek for a relation between the $r$-affine curvature $k$ and elementary affine curvature $\bar{k}$.

From the relations

$$
\begin{aligned}
\mathfrak{x}^{\prime} & =\frac{d \mathfrak{x}}{d \bar{S}} \frac{d \bar{S}}{d \bar{S}}=\frac{1}{\mathfrak{q}} \frac{d x}{d \bar{S}}, \\
x^{\prime \prime} & =\frac{1}{\mathfrak{q}^{2}} \frac{d^{2} x}{d \bar{S}^{2}}-\frac{1}{\mathfrak{q}^{3}} \frac{d \mathfrak{q}}{d \bar{S}} \frac{d \mathfrak{c}}{d \bar{S}}
\end{aligned}
$$

we have

and

$$
\xi_{2}=\mathfrak{q} \frac{d^{2} x}{d \bar{S}^{2}}-\frac{d \mathfrak{q}}{d \bar{S}} \frac{d x}{d \overline{\bar{S}}}
$$

$$
\xi_{2}^{\prime}=\frac{d^{3} r}{d \bar{S}^{3}}-\frac{1}{q} \frac{d^{2} q}{d \bar{S}^{2}} \frac{d x}{d \bar{S}}
$$

By means of (20) and the elementary formulae, which is found in case where $q=1$ in $\left.(20), \frac{d^{3} x}{d S^{3}}=-\bar{k} \frac{d r}{d s}{ }^{6}\right)$, we have

(6) W. Blaschke, loc. cit. p. 15. 


$$
k=q \bar{k}+\frac{d^{2} q}{d \bar{S}^{2}}
$$

From (19) and (21) we have

$$
\xi_{1}^{\prime \prime}=\frac{\xi_{2}^{\prime}}{\mathfrak{q}^{3}}-\frac{\mathfrak{q}^{\prime}}{\mathfrak{q}^{4}} \xi_{2},
$$

and

$$
\xi_{2}^{\prime \prime}=-k \xi_{1}^{\prime}-k^{\prime} \xi_{1}=-\frac{k}{\mathfrak{q}^{3}} \xi_{2}-k^{\prime} \xi_{1},
$$

consequently we obtain

$$
\xi_{1}^{\prime} \times \xi_{1}^{\prime \prime}=\frac{k}{q^{6}},
$$

and

$$
\xi_{2}^{\prime} \times \xi_{2}^{\prime \prime}=\frac{k^{2}}{q^{3}}
$$

Now we will proceed to seek for Bouquet's formulae: Let us take a point $P_{0}\left(x_{0}\right)$ on a curve $C$ as the origin and take the tangent and the $r$-affine normal as $x_{1}$ - and $x_{2}$-axis respectively. The coordinates $x(S)$ of a point $P$ sufficiently near to $P_{0}(S=0)$ can be expanded in power series of $S$, whose absolute value is sufficiently small:

$$
\mathfrak{x}(S)=\mathrm{x}_{0}(0)+\mathrm{x}_{0}^{\prime}(0) S+\frac{1}{2 !} \mathrm{x}_{0}^{\prime \prime}(0) S^{2}+\frac{1}{3 !} \mathrm{r}_{0}^{\prime \prime \prime}(0) S^{3}+\cdots \cdot
$$

Hence we have

$$
x_{0}=\{0,0\}, \quad r_{0}^{\prime}=\{1,0\}, \quad \chi_{0}^{\prime \prime}=\{0,1\},
$$

and from (15) and (20)

$$
x_{0}^{\prime \prime \prime}=\left\{-\frac{k_{0}}{q_{0}^{3}},-\frac{3 q_{0}^{\prime}}{q_{0}}\right\}
$$

Hence follow the extension of Bouquet's formulae:

$$
r_{1}(S)=S *-\frac{k_{0}}{3 ! q_{0}^{3}} S^{3}+\cdots,
$$

$$
\mathrm{x}_{2}(S)=*+\frac{1}{2} S^{2}-\frac{3 q_{0}^{\prime}}{3 ! q_{0}} S^{3}+\cdots
$$




\section{§. R-affine perpendicular distances.}

Take the point $T(3)$ and take a point $P(\mathfrak{x})$ on a curve $C$, then we define the r-affine perpendicular distance $\mathfrak{r}$ as follows,

$$
\mathfrak{r}=\mid \mathrm{r}-\jmath, \frac{d \mathrm{x}}{d S} .
$$

As a consequence of (3) and (5) we have

$$
\mathfrak{r}=\frac{1}{\mathfrak{q}} \mathfrak{x}-\mathfrak{z}, \frac{d \mathfrak{x}}{d \dot{S}} \mid=\frac{\mathfrak{p}}{\mathfrak{q}} .
$$

Now will prove the following.

Theorem 2. If a point 3 lies on a $r$-affine normal at $x$ on $C$, the $r$ affine perpendicular distance $\mathfrak{r}$ from $;$ to the tangent at $x$ has a stationary value.

Proof. By means of (32) and (33) we have

$$
\begin{aligned}
\mathfrak{r}= & \left|\begin{array}{l}
x_{1}-z_{1}, \frac{d x_{1}}{d S} \\
x_{2}-z_{2}, \frac{d x_{2}}{d S} \mid
\end{array}\right| \\
= & \left|\begin{array}{ll}
\left(s-\frac{k_{0}}{3 ! q_{0}^{3}} S^{3}+\cdots\right)-z_{1}, & 1-\frac{k_{0}}{2 q_{0}^{3}} S^{2}+\cdots \\
\left(\frac{1}{2} S^{2}-\frac{3 q_{0}^{\prime}}{3 ! q_{0}} S^{3}+\cdots\right)-z_{2}, \quad s-\frac{3 q_{0}^{\prime}}{2 q_{0}} S^{2}+\cdots
\end{array}\right| \\
= & -z_{1}\left(S-\frac{3 q_{0}^{\prime}}{2 q_{0}} S^{2}+\cdots\right)+z_{2}\left(1-\frac{k_{0}}{2 q_{0}^{3}} S^{2}+\cdots\right) \\
& +S^{2}\left(1-\frac{k_{0}}{3 ! q^{3}} S^{2}+\cdots\right)\left(1-\frac{3 q_{0}^{\prime}}{q_{0}} S+\cdots\right) \\
& -S^{2}\left(1-\frac{k_{0}}{2 ! q_{3}^{0}} S^{2}+\cdots\right)\left(\frac{1}{2} S-\frac{3 q_{0}^{\prime}}{3 ! q_{0}} S^{2}+\cdots\right),
\end{aligned}
$$

then

$$
\lim _{s \rightarrow 0} \frac{d \mathfrak{r}}{d S}=z_{1}=0 \quad \quad \text { (Q. E. D.) }
$$




\section{$\S 4$. Indicatrices of Tangents and of Curvatures.}

The indicatrix of tangents is defined as the curve $C_{1}$ described by the point $\xi_{1}$. Let us denote by $q_{1}$ the perpendicular distance from a point at the corresponding point on $E(\mathrm{e})$ for the point $\xi_{1}$ on $C_{1}$, then from (30) the $r$-arc length $S_{1}$ is given by the equation

$$
S_{1}=\int_{0}^{s} q_{1}\left(\xi_{1}^{\prime} \times \xi_{2}^{\prime \prime}\right)^{\frac{1}{3}} d S=\int_{0}^{s} \frac{\mathfrak{q}_{1}}{q^{2}} k^{\frac{1}{3}} d S .
$$

The indicatrix of curvatures is defined as the curve $C_{2}$ described by the point $-\xi_{2}$. Let us denote by $S_{2}$ the $r$-arc length on $C_{2}$, then from (31) $S_{2}$ is given by the equation

$$
S_{2}=\int_{0}^{s} \mathrm{a}\left(\left(-\xi_{2}^{\prime}\right) \times\left(-\xi_{2}^{\prime \prime}\right)\right)^{\frac{1}{3}} d S=\int_{0}^{s} k^{\frac{2}{3}} d S .
$$

Assume that $C$ is closed and denote by $L, L_{1}$ and $L_{2}$ respectively the perimeters of $C, C_{1}$ and $C_{2}$, and let us put

$$
\begin{array}{ll}
\text { Max. } k=\bar{k}, & \text { Min. } k=\underline{k}, \\
\text { Max. } \mathfrak{q}_{1} \mathfrak{q}^{-2}=\bar{Q} & \text { and Min. } \mathfrak{q}_{1} \mathfrak{q}^{-2}=\underline{Q},
\end{array}
$$

then we obtain the following inequalities

$$
\begin{aligned}
& Q k^{\frac{1}{3}} L<L_{1}<\bar{Q} \bar{k}^{\frac{1}{3}} L, \\
& \underline{k}^{\frac{2}{3}} L<L_{2}<\bar{k}^{\frac{2}{2}} L .
\end{aligned}
$$

Denote by $p_{2}$ and $p_{2}$ respectively the affine perpendicular distance and the elementary one from the origin $O$ to a point $-\xi_{2}$ on $C_{2}$, then we have

$$
p_{2}=\frac{\left|-\xi_{2},-\xi_{2}^{\prime}\right|}{\left|-\xi_{2}^{\prime}\right|}=\frac{|k|}{|k|\left|\xi_{1}\right|}=q \rho^{-\frac{1}{3}}
$$

therefore

$$
p=p \bar{p}^{-\frac{1}{3}}=p \frac{q}{p_{2}},
$$

that is

$$
\frac{p}{q}=\frac{p}{p_{2}} .
$$


Denote by $\bar{\rho}_{2}$ the radius of curvature at $-\xi_{2}$ on $C_{2}$, then by means of (20) and (31) we obtain

$$
\bar{\rho}_{2}=\frac{\left(\xi_{2}^{\prime \prime}\right)^{\frac{3}{2}}}{\left|\xi_{2}^{\prime} \times \xi_{2}^{\prime \prime}\right|}=\frac{\left|k^{3}\right|}{q^{3}} \bar{\rho} \cdot \frac{1}{\frac{\left|k^{2}\right|}{q^{3}}}=|k| \bar{\rho},
$$

therefore we have

$$
|k|=\frac{\bar{\rho}_{2}}{\bar{\rho}} .
$$

In consequence of (37) and (39) we have

$$
\mathfrak{p}_{2}=p_{1} \bar{\rho}_{2}^{\frac{1}{3}}=\mathfrak{q} \bar{\rho}^{-\frac{1}{3}}|k|^{\frac{1}{3}} \bar{\rho}^{\frac{1}{3}}=\mathfrak{q}|k|^{\frac{1}{3}},
$$

then

$$
|k|=\left(\frac{\mathfrak{b}_{2}}{\mathfrak{q}}\right)^{3} .
$$

Let us denote by $\mathfrak{p}_{0}$ and $\mathfrak{r}_{0}$ respectively the affine perpendicular distance and the $r$-affine perpendicular distance from the origin $O$ to a point $x$ on $C$. When $x_{0}$ is constant, the curve $C$ is called the relative affine circle.

If the curve $C$ be a $r$-affine circle, from (38) both curves $C$ and $C_{2}$ are similar and similarly situated, then from (40) the $r$-affine curvature $k$ is constant. Therefore from (42) it is concluded that the $C_{2}$ is a $r$ affine circle.

Conversely, if a curve $C$ be a r-affine circle, it is easily seen that the $r$-affine perpendicular distance from the origin to a point on $C$ is necessarily constant.

Hence we obtain the following

Theorem 3. A necessary and sufficient condition that a curve $C$ be a r-affine circle is that its $r$-affine curvature is constant.

Theorem 4. If a curve $C$ be a r-affine circle, its indicatrix of curvature is also a raffine circle.

In case where $q$ is equal to the unit length, by the relation $k=\bar{k}$ Theorem 2 is reduced to Theorem 1 in $\S 1$, because a conic is the curve whose affine curvature is constant. $\left({ }^{7}\right)$

Theorem 5. A necessary and sufficient condition that a curve $C$ be a conic is that its affine perpendicular distance from the properly chosen point is constant.

(7) W. Blaschke, Diff. Geom, II, p. 18. 
Now assume that $C$ is a $r$-affine circle, then by a symmetric transformation

$$
r=c^{\frac{3}{4}} x^{*}
$$

we have

$$
\mathfrak{r}^{*}=\frac{\mathfrak{p}^{*}}{\mathfrak{q}}=1
$$

that is

$$
\mathfrak{p}^{*}=\mathfrak{q} .
$$

Hereafter we consider the case where $\mathfrak{p}=\mathfrak{q}$ : Denote by $\xi$ the elementary normal unit vector, and let us put

$$
\begin{array}{ll}
p=(-\mathfrak{r} \xi), & \bar{p}_{2}=(\dddot{x} \xi), \\
q=(-\mathfrak{e} \xi), & \bar{q}_{2}=(\ddot{e} \xi),
\end{array}
$$

where $\ddot{x}$ and $\ddot{e}$ mean respectively derivatives with respect to the elementary affine arc length.

Then from (45) we have

$$
\frac{p}{\bar{p}_{2}}=\frac{q}{\bar{q}_{2}} \cdot\left(^{8}\right)
$$

Let us put

$$
\frac{p}{\bar{p}_{2}}=\frac{q}{\bar{q}_{2}}=\lambda,
$$

then we have

$$
r+\lambda \ddot{x}=\mu \dot{x} .
$$

By differentiating (49) with respect to $\bar{S}$ we obtain

then

$$
\dot{r}-\lambda \bar{k} \dot{x}+\dot{\lambda} \ddot{x}=\dot{\mu} \dot{x}+\mu \ddot{x},
$$

$$
\mu=\dot{\lambda} \quad \text { and } \quad \frac{1}{\lambda}-\frac{\ddot{\lambda}}{\lambda}=\bar{k},
$$

consequently (49) becomes

$$
x+\lambda \ddot{x}=\dot{\lambda} \dot{x} .
$$

(8) This is easily seen from (3) in this paper and (153) in W. Blaschke, loc, cit., p. 32 . 
Differentiating (7) with respect to $\bar{S}$ we have

$$
\ddot{\mathrm{q}}=\dot{\mathrm{S}}^{-\frac{1}{2}} \ddot{\mathrm{e}}+\frac{1}{2} \ddot{\mathrm{S}}^{-\frac{3}{2}} \dot{\mathrm{e}},
$$

where

$$
\ddot{\mathfrak{x}}=\frac{d^{2} x}{d \bar{S}^{2}}, \ddot{e}=\frac{d^{2} e}{d \bar{s}^{2}}, \dot{\mathfrak{e}}=\frac{d e}{d \bar{s}}, \ddot{\bar{S}}=\frac{d^{2} \bar{S}}{d \bar{s}^{2}} \text { and } \dot{\bar{S}}=\frac{d \bar{S}}{d \bar{s}} \text {. }
$$

Substituting in (51) we obtain

$$
x+\lambda\left(\dot{S}^{-\frac{1}{2}} \ddot{e}+\frac{1}{2} \ddot{\bar{S}} \dot{S}^{-\frac{3}{2}} \dot{e}\right)-\dot{\lambda}^{-\frac{1}{2}} \dot{e}=0 .
$$

From (48) in the same manner as we have obtained (51) we have the equation

$$
e+\lambda \ddot{e}=\dot{\lambda} \dot{e} .
$$

By means of (53) the equation (52) becomes

$$
x-\dot{\bar{S}}^{-\frac{1}{2}} \mathrm{e}+\frac{1}{2} \lambda \ddot{\bar{S}} \dot{\bar{S}}^{-\frac{3}{2}} \dot{\mathrm{e}}=0
$$

Differentiating (54) with respect to $\bar{s}$, we have

$$
\dot{S}^{\frac{3}{2}} \dot{e}-\dot{S}^{-\frac{1}{2}} \dot{e}+\frac{1}{2} \lambda \ddot{S} \dot{S}^{-\frac{3}{2}} \ddot{e}+\frac{1}{2} \frac{d}{d \dot{s}}\left(\lambda \ddot{S} \dot{S}^{-\frac{3}{2}}\right) \dot{e}=0
$$

and by substituting (53)

$$
\left\{\dot{S}^{\frac{3}{2}}-\dot{\bar{S}}^{-\frac{1}{2}}+\frac{1}{2} \dot{\lambda} \ddot{\bar{S}} \dot{\bar{S}}^{-\frac{3}{2}}+\frac{1}{2} \frac{d}{d \bar{S}}\left(\lambda \ddot{\bar{S}} \dot{\bar{S}}^{-\frac{3}{2}}\right)\right\} \dot{\mathrm{e}}=0
$$

Hence the affine arc length $\bar{S}$ must satisfy the following differential equation

$$
\dot{S}^{4}-\dot{S}^{2}+\lambda \ddot{\vec{S}} \dot{S}+\frac{1}{2} \lambda \ddot{S} \dot{S}-\frac{3}{4} \lambda \dot{S}^{2}=0
$$

From the relation $q / \bar{q}_{2}=\lambda$ in (48), $\lambda$ is found as a function of the affine arc length $\bar{s}$ of the unit curve, and consequently if we define $\bar{S}$ as a function of $\overline{\bar{s}}$ such that it satisfies the differential equation (55), the unit $r$-affine circle is determined as the solution of the differential equation (51). Thus we have the following

Theorem 6. If the affine arc length $\bar{S}$ of a curve satisfies (55) and its radius vector $x$ of the point on the curve satisfies (51), the curve necessarily be an unit r-affine circle, and its converse is also true. And the 
$r$-affine circle is a curve such that it is reduced to an unit r-affine circle by a symmetric transformation.

\section{$\S 5$. R-affine evolutes.}

$R$-affine normals of a curve $C(x)$ envelope a curve $C^{*}\left(r^{*}\right)$ which is called the $r$-affine evolute. A point $\mathrm{r}^{*}$ on $C^{*}$ is given as follows

$$
x^{*}=x+\lambda \xi_{2} .
$$

Differentiating (56) with respect to $S$ we have

$$
\frac{d x^{*}}{d S}=\left(1-\lambda k ; \xi_{1}+\frac{d \lambda}{d S} \xi_{2} \equiv \sigma \xi_{2},\right.
$$

then

$$
\lambda=\frac{1}{k}
$$

therefore (56) becomes

$$
x^{*}=x+\frac{1}{k} \xi_{2} .
$$

The point $x^{*}$ is called the centre of r-affine curvature at a point to on $C$.

Denote by $\mathfrak{p}^{*}$ and $\mathfrak{r}^{*}$ respectively the affine perpendicular distance and $r$-affine perpendicular distance from $x^{* *}$ to $x$, then from (33) we obtain

$$
\mathfrak{r}^{*}=\left|r-r^{*}, \frac{d x}{d S}=\right|-\frac{1}{k} \xi_{2}, \xi_{1} \mid=\frac{1}{k} \text {. }
$$

Hence we obtain from (58) and (57):

Theorem 7. A necessary and sufficient condition that a curve be a $r$-affine circle is that the r-affine normals pass through a fixed point.

Denote by $S^{*}$ the $r$-affine are length on the evolute $x^{*}$ and denote by $q^{*}$ the affine distance at the corresponding point on $E$, then we obtain

$$
\frac{d S^{*}}{d S}=q^{*} \frac{k^{\prime \frac{2}{3}}}{k}
$$

\section{§6. Natural equations.}

Let $C$ and $C^{*}$ be two curves defined in terms of their respective $r$-affine arc length $S$, and let us make correspond points with the same 
values of $S$ on each curve. Let us assume, furthermore, that at corresponding points the $r$-affine curvature have the same value. We shall prove that $C$ and $C^{*}$ are affinely transformable to each other by the same method as in the proof given by Prof. $W$. Blaschke. $\left({ }^{9}\right)$

From the assumption $x$ and $\mathfrak{c}^{*}$ must satisfy the differential equations (20)

or

$$
\xi_{2}^{\prime}+k(S) \xi_{1}=0
$$

$$
\left(q^{3} x^{\prime \prime}\right)^{\prime}+k(S) x^{\prime}=0
$$

At first we consider the case where both tangents at the corresponding points $x$ and $x^{*}$ are parallel. In this case $x_{1}^{\prime}$ and $x_{2}{ }^{\prime}$ must be a system of solutions of the linear differential equation of the second order.

$$
\left(q^{3} y^{\prime}\right)^{\prime}+k y=0
$$

Take $y_{1}$ and $y_{2}$ as the linearly independent solutions of (61), then we have

and consequently

$$
\left(q^{3} y_{2}^{\prime}\right)^{\prime} y_{1}-\left(q^{3} y_{1}^{\prime}\right)^{\prime} y_{2}=0
$$

$$
\left(q^{3} y_{2}^{\prime}\right)^{\prime} y_{1}-\left(q^{3} y_{1}^{\prime}\right) y_{2}=\text { constant }
$$

By multiplication of a suitable constant we can make the above constant equal to one so that

Now let us put

$$
\left(q^{3} y_{2}^{\prime}\right) y_{1}-\left(q^{3} y_{1}^{\prime} y_{2}=1\right.
$$

$$
\begin{aligned}
& x_{1}^{\prime}=a_{11} y_{1}+a_{12} y_{2}, \\
& x_{2}^{\prime}=a_{21} y_{1}+a_{22} y_{2} .
\end{aligned}
$$

Then we have

$$
\left(\mathfrak{c}^{\prime}, \mathfrak{x}^{\prime \prime}\right)=\left(a_{11} a_{22}-a_{12} a_{21}\right)\left(y_{1} y_{2}^{\prime}-y_{2} y_{1}^{\prime}\right)=\frac{a}{\mathfrak{q}^{3}} \cdot \quad\left(\left|a_{i:}\right| \equiv a \neq 0\right)
$$

Let us take $a=1$ and put

$$
x_{1}^{*}=\int_{0}^{s} y_{1} d S, \quad x_{1}^{*}=\int_{0}^{s} y_{2} d S
$$

thus the general solutions of (60) are given by the following equations

$$
\begin{aligned}
& x_{1}=a_{10}+a_{11} x_{1}^{*}+a_{12} x_{2}^{*}, \\
& x_{2}=a_{20}+a_{21} x_{1}^{*}+a_{22} x_{2}^{*},
\end{aligned}
$$

(9) Loc. cit, p. 17. 
where

$$
\left(x^{\prime}, q^{3} x^{\prime \prime}\right)=1
$$

In case where both tangents at the corresponding point $x$ and $x^{*}$ are not parallel, if $q^{*}$ be affinely equivalent to $q$, the curve must be a conic or an affine transform of a periodic curve. $\left({ }^{10}\right)$ In this case we can make the corresponding tangents parallel by an equivalent affine transformation, consequently it is reduced to the preceding case.

Hence we have the following

Theorem 8. If the r-affine curvature $k$ is given as an analytic function of the r-affine arc length $S$, a curve whose $r$-affine curvature is $k(S)$ is uniquely determined in general, except for the translation. In case where the unit curve is a periodic curve such that $\mathrm{q}^{*}=\mathfrak{q}$, the curve is uniquely determined except for translations and an equivalent affine transformation, and in case where the unit curve is a conic the curve is uniquely determind except for translations and equivalent affine transformations.

We call $k=k(S)$ the natural equation.

\section{\$7. Curves of constant r-affine breadth.}

I have made an investigation concerning the affine breadth.( $\left.{ }^{11}\right) \mathrm{Re}$ cently $L$. Beretta and $A$. Maxia $\left({ }^{12}\right)$ have made a study of the same subject in his paper. I will here extend the affine breadth in our geometry: Take a convex closed plane curve $C(x)$ and denote the points $P_{1}$ and $P_{2}$ such that the trangent lines at $P_{1}$ and $P_{2}$ are parallel to each other by $x_{1}$ and $x_{2}$. Then the $r$-affine breadth $\mathfrak{b}$ is defined as follows

$$
\mathfrak{b}=2 \frac{\left(\mathfrak{x}_{2}-\mathfrak{x}_{1}\right) \xi_{1}}{\left(\mathfrak{l}_{1} \xi_{1}\right)+\left(\mathfrak{y}_{2} \xi_{2}\right)}
$$

where $\xi_{i}$ are the unit normal vectors, at $\mathfrak{x}_{i}$ and $\mathfrak{y}_{i}$ denotes the $r$-affine normal vector at $x_{i}$.

From the definition we have the following theorems:(13)

Theorem 9. Let us denote by $\mathfrak{1}_{i}$ the $r$-affine perpendicular distance from $\mathfrak{x}_{i}$ to $\mathfrak{x}_{k}(i, k=1,2 ; i \neq k)$, where $x_{1}$ and $\mathfrak{x}_{2}$ are the points of contact of the parallel tangents, then $\mathfrak{b}$ is equal to the harmonic mean of $t_{1}$ and $t_{2}$ and $\mathfrak{b}$ is an affine invariant.

(10) J. Hirakawa, E. R. G., I, loc. cit., Theorem 2 in $\& 2$.

(11) J. Hirakawa, The affine breadth and its relation to the relative breadth, Jap. Journ. of Math., XII (1935).

(12) L. Beretta e A. Maxia, Insiemi convessi e orbiformi, Memoria estratta dai Rendiconti dl Matematica e delle sue appliazioni, Serie V. Volume 1-Fascicolo 1-1940.

(13) We can prove in the same way as in the preceding literature (11). 
Theorem 10. A necessary and sufficient condition that the r-affine breadth of $x$ be constant is that the two vectors $\mathfrak{l}_{2}$ and $\mathfrak{y}_{1}-\mathfrak{y}_{2}$ are parallel.

Theorem 11. If $\mathfrak{b}$ is constant, then the following relation holds

$$
\mathfrak{b}=2 \frac{\tilde{\rho}_{1}+\tilde{f}_{2}}{k_{1} \bar{\rho}_{1}+k_{2} \tilde{p}_{2}},
$$

where $k_{i}$ denotes the $r$-affine curvature at $\mathrm{r}_{i}$.

Theorem 12. If the r-affine normals at the points of contact of two parallel tangents are r-affine double normal of an oral, the oval has the following properties:

$1^{\circ}$. The oval is a curve of constant $r$-affine breadth.

$2^{\circ}$. The sum of two radii of $r$-affine curvature at $\mathfrak{x}_{1}$ and $\mathrm{r}_{2}$ is constant $(=\mathfrak{b})$.

$3^{\circ}$. The indicatrix of $r$-affine curvature is central.

$4^{\circ}$. The r-affine perpendicular distance between two points $\mathfrak{x}_{1}$ and $\mathfrak{r}_{2}$ is constant $(=\mathfrak{b})$.

$5^{\circ}$. The sum of the two $r$-affine perpendicular distances from the origin to $\mathrm{x}_{1}$ and $\mathrm{r}_{2}$ is constant $(=\mathfrak{b})$.

Proof. From the assumption it can be put

$$
\mathfrak{y}_{2}=-\sigma \mathfrak{l}_{1},
$$

where $\sigma$ is a positive scalar quantity.

Then we have

$$
-k_{2} x_{2}^{\prime}=\sigma k_{1} x_{1}^{\prime}-\sigma^{\prime} \mathfrak{y}_{1}
$$

consequently $\sigma^{\prime}=0$ or $\sigma=$ constant.

According to (64) we have $\mathfrak{y}_{1}=-\sigma \mathfrak{y}_{2}$, therefore $\sigma=1$. then

$$
\mathfrak{y}_{1}+\mathfrak{y}_{2}=0 \text {. }
$$

Hence we conclude that the indicatrix of $r$-affine curvature is central.

From the assumption and Theorem 10 it is evident that the $r$-affine breadth $\mathfrak{b}$ is constant.

By the definition of the $r$-affine breadth and the assumption it can be put

$$
\mathfrak{r}_{1}-\mathfrak{c}_{2}+\frac{\mathfrak{b}}{2}\left(\mathfrak{y}_{1}-\mathfrak{y}_{2}\right)=0
$$

From (66) we have

$$
\mathfrak{x}_{1}-\mathfrak{x}_{2}=-\mathfrak{b} \mathfrak{y}_{1}
$$


Let us form the vector product of both members of the above equation with $x_{1}^{\prime}$, then from $\left|\mathfrak{x}_{1}^{\prime}, \mathfrak{y}_{1}\right|=1$ we have

$$
\left|r_{1}^{\prime}, r_{1}-r_{2}\right|=-\mathfrak{b},
$$

consequently

$$
\left|\mathfrak{x}_{1}, x_{1}^{\prime}\right|+\left|\mathfrak{x}_{2}, x_{2}^{\prime}\right| \lambda=\mathfrak{b}
$$

Let $\mathfrak{r}_{i}$ denote the $r$-affine perpendicular distance from the origin to $\mathfrak{r}_{i}$, then (70) becomes

$$
\mathfrak{r}_{1}+\mathfrak{r}_{2} \lambda=\mathfrak{b}
$$

consequently in the same manner we obtain

$$
\mathfrak{r}_{2}+\mathfrak{r}_{1} \frac{1}{\lambda}=\mathfrak{b} \text {. }
$$

By means of (70) and (71) we have $\lambda=1$, then

$$
r_{1}^{\prime}=-r_{2}^{\prime} \text {. }
$$

Therefore we obtain

$$
\mathfrak{r}_{1}+\mathfrak{r}_{2}=\mathfrak{b}=\text { constant } .
$$

By means of (69) and (73) we have

$$
\mathrm{t}_{1}=\left|\mathrm{x}_{1}-\mathrm{x}_{2}, \mathrm{x}_{1}^{\prime}\right|=\left|\mathfrak{x}_{2}-\mathrm{r}_{1}, \mathfrak{x}_{2}^{\prime}\right|=\mathrm{t}_{2}=\mathfrak{b}=\text { constant } .
$$

Differentiating (66) with respect to $S_{1}$, we have

$$
\mathfrak{y}_{1}^{\prime}+\mathfrak{y}_{2}^{\prime} \frac{d S_{2}}{d S_{1}}=0
$$

from (20)

$$
-k_{1} \mathfrak{x}_{1}^{\prime}-k_{2} x_{2}^{\prime} \frac{d S_{2}}{d S_{1}}=0
$$

then

$$
\frac{k_{1}}{k_{2}}=\frac{d S_{2}}{d S_{1}}
$$

Differentiating (68) with respect to $S_{1}$ and substituting (76), we have

$$
\mathfrak{b}=\frac{1}{k_{1}}+\frac{1}{k_{2}} \text {. }
$$




\section{Chapter II. Theory of space curves.}

\section{$\S 1$. Affine perpendicular distances.}

Take a point $P(x(t))$ on a space curve $C$ and take three consecutive points $P^{\prime}\left(\mathfrak{x}\left(t_{1}\right)\right), P^{\prime \prime}\left(\mathfrak{x}\left(t_{2}\right)\right)$ and $P^{\prime \prime \prime}\left(\mathfrak{c}\left(t_{3}\right)\right)$ on it, where $t_{1}=t+\Delta t, t_{2}=t_{1}+\Delta t_{1}$ and $t_{3}=t_{2}+\Delta t_{2}$.

Let $A(\mathfrak{a})$ be a fixed point in space, and denote by $V_{1}$ and $V_{2}$ respectively the volumes of two tetrahedrons $A-P_{1} P_{2} P_{3}$ and $P-P_{1} P_{2} P_{3}$, then we consider the following quantities:

(1) $\Omega=\lim _{\Delta t \rightarrow 0} \frac{6 V_{1}}{\Delta t^{3}}$

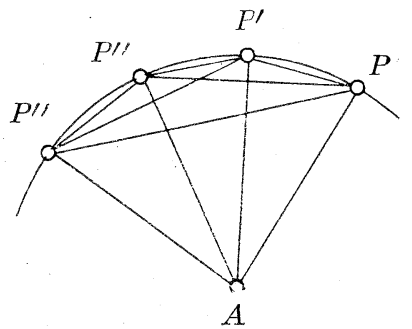

$$
\begin{aligned}
& =\lim _{\Delta t \rightarrow 0} \frac{1}{\Delta t^{3}}\left|\mathfrak{a}-\mathfrak{r}\left(t_{1}\right), \mathfrak{r}\left(t_{1}+\Delta t_{1}\right)-\mathfrak{r}\left(t_{1}\right), \mathfrak{r}\left(t_{2}+\Delta t_{2}\right)-\mathfrak{r}\left(t_{2}\right)\right| \\
& =\lim _{\Delta t \rightarrow 0} \frac{1}{\Delta t^{3}}\left|\mathfrak{a}-\mathfrak{r}\left(t_{1}\right), \dot{\mathfrak{r}}\left(t_{1}\right) \Delta t_{1}+\frac{\ddot{x}\left(t_{1}\right)}{2 !} \Delta t_{1}^{2}+\cdots, \dot{\dot{r}}\left(t_{2}\right) \Delta t_{2}+\frac{\ddot{x}\left(t_{2}\right)}{2 !} \Delta t_{2}^{2}+\cdots\right| \\
& =|\mathfrak{a}-\mathfrak{r}, \dot{x}, \ddot{x}| \text {, }
\end{aligned}
$$

and

( 2 )

$$
\begin{aligned}
& \Sigma^{2}=\lim _{\Delta t \rightarrow 0} \frac{6 V_{2}}{\Delta t^{6}} \\
& =\lim _{\Delta t \rightarrow 0} \frac{1}{\Delta t^{6}}\left|\mathfrak{r}(t+\Delta t)-\mathfrak{r}(t), r\left(t_{1}+\Delta t_{1}\right)-r\left(t_{1}\right), \mathfrak{g}\left(t_{2}+\Delta t_{2}\right)-\mathfrak{r}\left(t_{2}\right)\right| \\
& =|\dot{x}, \ddot{x}, \ddot{x}| \text {. }
\end{aligned}
$$

Let us put

$$
\mathfrak{p}_{1}=\frac{\Omega}{\Sigma}
$$

then we have

(4) $\quad \mathfrak{p}_{1}=\frac{|a-x, \dot{x}, \ddot{x}|}{|\dot{x}, \ddot{x}, \ddot{x}|^{\frac{1}{2}}}=\frac{\left|a-x, d x, d^{2} r\right|}{(d \bar{S})^{3}}=\left|a-r, \frac{d x}{d \bar{S}}, \frac{d^{2} x}{d \bar{S}^{2}}\right|$,

where $d \bar{S}$ denotes the affine linear element.

$p_{1}$ is called the affine perpendicular distance from $A$ to the osculating plane at a point $x$ on $C$.

It is evident that $\mathfrak{p}_{1}$ is an affine invariant and an invariant for parameter-transformations. 
Let us denote the elementary perpendicular distance by $p_{1}$, and denote the radius of curvature by $\rho$ and denote the radius of torsion by $\tau$, then we have

$$
\left|p_{1}\right|=p_{1} \rho^{-1}|\tau|^{-\frac{1}{2}} .
$$

Now we consider the affine perpendicular distance $\rho_{1}$ concerning an asymptotic curve $C$ on a suface $F(x(u, v))$. Take the asymptotic lines as parameteric curves, and let $C$ be a parameteric curve $v=$ constant, then we have

$$
\begin{aligned}
& \mathfrak{x}_{u u}=\alpha \mathfrak{x}_{u}+\beta \mathfrak{x}_{v}, \\
& \mathfrak{x}_{v v}=\gamma \mathfrak{x}_{u}+\delta \mathfrak{x}_{v},
\end{aligned}
$$

and then

$$
\begin{aligned}
\mathfrak{x}_{u u u} & =\alpha \mathfrak{x}_{u u}+\beta \mathfrak{x}_{u v}+\alpha_{u} \mathfrak{x}_{u}+\beta_{u} \mathfrak{x}_{v} \\
& =\alpha\left(\alpha \mathfrak{x}_{u}+\beta \mathfrak{x}_{v}\right)+\alpha_{u} \mathfrak{x}_{u}+\beta_{u} \mathfrak{x}_{v}+\beta \mathfrak{x}_{u v} \\
& =\left(a^{2}+\alpha_{u}\right) \mathfrak{x}_{u}+\left(\alpha \beta+\beta_{u}\right) \mathfrak{x}_{v}+\beta \mathfrak{x}_{u v} .
\end{aligned}
$$

Therefore, from (4), (6) and (7), we obtain

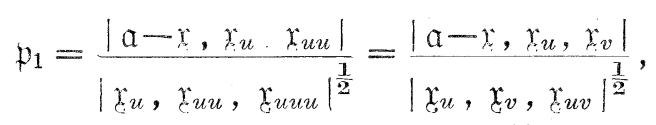

then it is seen that $\rho_{1}$ is equal to "Affinentfernung" $\left({ }^{14}\right)$ defined by Prof. W. Blaschke.

Consequently a geometrical meaning of the affine perpendicular distance $p_{1}$ from a point $A$ to a point $P$ on a surface $F$ is given as follows :

Theorem 1. If we denote by $V_{2}$ and $V_{1}$ respectively the volumes of the tetrahedron formed by the point $P$ and the three consecutive points on an asymptotic line passing through $P$ and the tetrahedron formed by the point $A$ and the same three consecutive points of $P$, the affine perpendicular distance $\mathfrak{p}$ from $A$ to $P$ is equal to $\lim _{\Delta t \rightarrow 0} \sqrt{6} V_{1} / \sqrt{V_{2}}$.

Now let us put

$$
\mathfrak{k}_{2}=\left|\mathfrak{x}-\mathfrak{a}, \frac{d x}{d \overline{\bar{S}}}, \frac{d^{3} x}{d \bar{S}^{3}}\right|,
$$

(14) Loc. cit, Diff. Geom. II., p. 110. 
then we call $\mathfrak{p}_{2}$ the affine perpendicular distance from a point a to the affine rectifying plane at a point $\mathfrak{x}$ on $C$. Hence. $\mathfrak{p}_{2}$ is evidently an affine invariant.

\section{§. R-affine arc length.}

Take a closed convex surface $E(\mathrm{e})$ as the unit surface and let the point $P(x)$ on a space curve $C$ so correspond to the point $Q(e)$ on the unit surface $E$ that the tangent plane at $Q$ is parallel to the affine rectifying plane at $P$ on $C$. The locus of $Q$ is called the unit curve, $C_{0}(\mathrm{e})$. Let us denote the affine perpendicular distance from the origin to $Q$ on $E$ by $q$, then we have

$$
q=\frac{\left|e_{u}, e_{v},-e\right|}{\left|l n-m^{2}\right|^{\frac{1}{4}}},
$$

where $\quad l=\left|\mathfrak{e}_{u}, \mathfrak{e}_{v}, \mathfrak{e}_{u u}\right|, m=\left|\mathfrak{e}_{u}, \mathfrak{e}_{v}, \mathfrak{e}_{u v}\right|$ and $n=\left|\mathfrak{e}_{u}, \mathfrak{e}_{v}, \mathfrak{e}_{v v}\right|$.

The relative affine arc lengtn $S$ is defined as

$$
d S=\mathfrak{q} \Sigma^{\Sigma^{\frac{x}{3}}} d t=\mathfrak{q}|\dot{x}, \ddot{x}, \ddot{x}|^{\frac{1}{6}} d t=\mathfrak{q} d \bar{S} .
$$

If $t=S$, we have

$$
q^{6}\left|x^{\prime}, x^{\prime \prime}, x^{\prime \prime \prime}\right|=1
$$

Now let up put

$$
\mathfrak{x}^{\prime}=\xi_{1}, \quad \mathfrak{q}^{\prime \prime}=\xi_{2}, \quad q^{6} \mathfrak{r}^{\prime \prime \prime}=\xi_{3},
$$

then we get

$$
\left|\xi_{1}, \xi_{2}, \xi_{3}\right|=1
$$

and consequently

$$
\left|\xi_{1}, \xi_{2}, \xi_{3}^{\prime}\right|=0,
$$

Therefore we have the fundamental equation:

$$
\xi_{3}^{\prime}+k_{1} \xi_{2}+k_{2} \xi_{1}=0,
$$

where $k_{1}$ and $k_{2}$ are respectively called the relative affine curvature and the relative affine torsion. Moreover $\xi_{1}, \xi_{2}$ and $\xi_{3}$ are called respectively the tangential, principal normal- and binormal-unit vector. Hence we have the extension of Frenet-Serret formulae: 


$$
\xi_{1}^{\prime}=\xi_{2}, \quad \xi_{2}^{\prime}=\frac{\xi_{2}}{\mathfrak{q}^{6}}, \quad \xi_{3}^{\prime}=-k_{1} \xi_{2}-k_{2} \xi_{1} .
$$

$k_{1}$ and $k_{2}$ are found from the following

$$
k_{1}=\left|\xi_{1}, \xi_{3}, \xi_{3}^{\prime}\right|, \quad k_{2}=-\left|\xi_{2}, \xi_{3}, \xi_{3}^{\prime}\right| .
$$

In the next place we will proceed to seek for Bouquet's formulae: Let us take a point $P_{0}\left(\mathrm{x}_{0}\right)$ on a curve $C$ as the origin and take the tangent, the $r$-affine principal normal and the $r$-affine binormal respectively as $x_{1}-, x_{2}-$ and $x_{3}$-axis. The coordinates $x(S)$ of a point $P$ sufficiently near to $P_{0}(S=0)$ can be expanded in power series of $S$, whose absolute value is sufficiently small.

$$
r(S)=x_{0}^{\prime}+x_{0}^{\prime} S+\frac{x_{0}^{\prime \prime}}{2 !} S^{2}+\cdots
$$

However we find

$$
r_{0}=\{0,0,0\}, \quad x_{0}^{\prime}=\{1,0,0\}, \quad x_{0}^{\prime \prime}=\{0,1,0\}, \quad r_{0}^{\prime \prime \prime}=\{0,0,1\},
$$

and Ifrom (12)

$$
\begin{aligned}
\chi_{0}^{\mathrm{iV}}= & \left\{-\frac{k_{20}}{Q_{0}},-\frac{k_{10}}{Q_{0}},-\frac{Q_{0}^{\prime}}{Q_{0}^{2}}\right\}, \\
\dot{\mathrm{c}}_{0} \mathrm{~V}= & \left\{\frac{1}{Q^{2}}\left(2 Q_{0}^{\prime} k_{20}-Q_{0} k_{20}^{\prime}\right), \frac{1}{Q_{v}^{2}}\left(2 k_{10} Q_{0}^{\prime}-Q_{0} k_{10}^{\prime}-Q_{0} k_{20}\right),\right. \\
& \left.\frac{1}{Q_{0}^{3}}\left(2 Q_{0}^{\prime}-Q_{0} Q_{0}^{\prime \prime}-Q_{0} k_{10}\right)\right\},
\end{aligned}
$$

where $k_{10}, k_{20}$ and $Q_{0}=q_{0}^{6}$ denote respectively the values of corresponding quantities $k_{1}, k_{2}$ and $Q=q^{6}$ for $S=0$.

Thus we get the extension of Bouquet's formulae:

$$
\begin{aligned}
x_{1}(S) & =S * *-\frac{k_{20}}{4 ! Q_{0}} S^{4}+\frac{1}{5 ! Q_{0}^{2}}\left(2 Q_{0}^{\prime} k_{20}-Q_{0} k_{20}^{\prime}\right) S^{5}+\cdots, \\
\text { (19) } x_{2}(S) & =* \frac{1}{2 !} S^{2} *-\frac{k_{10}}{4 ! Q_{0}} S^{4}+\frac{1}{5 ! Q_{0}^{2}}\left(2 k_{00} Q_{0}^{\prime}-Q_{0} k_{10}^{\prime}-Q_{0} k_{20}\right) S^{5}+\cdots, \\
x_{3}(S) & =* \frac{1}{3 ! Q_{0}^{2}} S^{3}-4 ! Q_{0}^{\prime} S^{4}+\frac{1}{5 ! Q^{3}}\left(2 Q_{0}^{\prime 2}-Q_{0} Q_{0}^{\prime \prime}-Q_{0} k_{10}\right) S^{5}+\cdots
\end{aligned}
$$


Now from our correspondence between two points $x$ and $\mathfrak{e}$ we can put

$$
\mathfrak{e}_{i}=a_{i}^{1} \bar{\xi}_{1}+a_{i}^{3} \bar{\xi}_{3}, \quad(i=1,2)
$$

where $e$ is the radius vector of the point on the unit curve $C_{0}$ and $\mathfrak{e}_{i}=\frac{\partial c}{\partial u^{i}}$. Then e can be considered as a function of $\bar{S}$. Consequently differentiating (20) with respect to $\bar{S}$ we have

$$
\begin{aligned}
\mathfrak{e}_{i j} \frac{d \bar{u}^{j}}{d \bar{S}} & =\frac{d a_{i}^{1}}{d \bar{S}} \bar{\xi}_{1}+\frac{d a_{i}^{3}}{d \bar{S}} \bar{\xi}_{3}+a_{i}^{1} \bar{\xi}_{2}-a_{i}^{3}\left(\bar{k}_{1} \bar{\xi}_{2}+\bar{k}_{2} \bar{\xi}_{1}\right) \\
& =\left(\frac{d a_{i}^{1}}{d \bar{S}}-a_{i}^{3} \bar{k}_{2}\right) \bar{\xi}_{1}+\left(a_{\imath}^{1}-\bar{k}_{1} a_{i}^{3}\right) \bar{\xi}_{2}+\frac{d a_{i}^{3}}{d \bar{S}} \bar{\xi}_{3},
\end{aligned}
$$

then

$$
l_{i j} \frac{d u^{j}}{d \bar{S}}=\left|\mathfrak{e}_{1}, \mathfrak{e}_{2}, \mathfrak{e}_{i j} \frac{d u^{j}}{d \bar{S}}\right|=a\left(\pi_{1} a_{i}^{3}-a_{i}^{1}\right)
$$

and

$$
\frac{d u^{j}}{d \bar{S}}=a\left(\bar{k}_{1} \alpha_{i}^{3}-a_{i}^{1}\right) l^{i j}
$$
obtain

If we take the asymptotic curves as parameteric curves, then we

$$
\begin{aligned}
& \frac{d u^{1}}{d \bar{S}}=a\left(\bar{k}_{1} a_{2}^{3}-a_{2}^{1}\right) l^{21}=\frac{1}{l_{12}}\left(\bar{k}_{1} a_{2}^{3}-a_{2}^{1}\right), \\
& \frac{d u^{2}}{d \bar{S}}=a\left(\bar{k}_{1} a_{1}^{3}-a_{1}^{1}\right) l^{12}=\frac{1}{l_{12}}\left(\bar{k}_{1} a_{1}^{3}-a_{1}^{1}\right) .
\end{aligned}
$$

\section{\$. R-affine perpendicular distances.}

The $r$-affine perpendicular distance $\mathfrak{r}_{1}$, from a point $z$ to the osculating plane at $x$ is defined as (3) in $\$ 1$ by the equation

$$
\mathfrak{r}_{1}=q^{2}\left|z-r, \frac{d \mathfrak{r}}{d S}, \frac{d^{2} x}{d S^{2}}\right| \text {. }
$$


Thus we obtain easily the following equation

$$
\mathfrak{r}_{1}=\frac{\mathfrak{p}_{1}}{\mathfrak{q}}
$$

In the next place the $r$-affine perpendicular distance $\mathfrak{r}_{2}$ from a point $z$ to the rectifying plane at $x$ is defined analogous to (9) by the expression

$$
\mathfrak{r}_{2}=\left|x-z, \frac{d x}{d S}, \frac{d^{3} x}{d S^{3}}\right| q^{6}
$$

From the definition we have

$$
\mathfrak{r}_{2}=(x-z)\left(\xi_{1} \times \xi_{3}\right)=\left|x-z, \xi_{1}, \xi_{3}\right|
$$

By means of the moving trihedral $(x)-\left(\xi_{1}\right)\left(\xi_{2}\right)\left(\xi_{3}\right)$ any point $z$ in space can be represented by

$$
z=\mathfrak{x}+a_{1} \xi_{1}+a_{2} \xi_{2}+a_{3} \xi_{3} .
$$

Substituting in (25) we have

$$
\mathfrak{r}_{1}=a_{3}
$$

Substituting in (27) we have

$$
\mathrm{r}_{2}=a_{2}
$$

Now we prove that if a point $z$ lies on the $r$-affine rectifying plane of a space curve, then the $r$-affine perpendicular distance is stationary.

By means of (19) and (25) we obtain

$$
\mathfrak{x}_{1}=q^{2}\left|\begin{array}{ccc}
z_{1}-\left(S-\frac{k_{20}}{4 ! Q_{0}} S^{4}+\cdots\right) & 1-\frac{k_{20}}{3 ! Q_{0}} S^{3}+\cdots & -\frac{k_{20}}{2 Q_{0}} S^{2}+\cdots \\
z_{2}-\left(\frac{1}{2 !} S^{2}-\frac{k_{10}}{4 ! Q_{0}} S^{4}+\cdots\right) & S-\frac{k_{10}}{3 ! Q_{0}} S^{3}+\cdots & 1-\frac{k_{10}}{2 Q_{0}} S^{2}+\cdots \\
z_{3}-\left(\frac{1}{3 ! Q_{0}} S^{3}-\frac{Q_{0}^{\prime}}{4 ! Q_{0}^{2}} S^{4}+\cdots\right) & \frac{1}{2 Q_{0}} S^{2}-\frac{Q_{0}^{\prime}}{3 ! Q_{0}^{\prime}} S^{3}+\cdots, \frac{1}{Q_{0}} S-\frac{Q_{0}^{\prime}}{2 Q_{0}^{2}} S^{2}+\cdots
\end{array}\right|,
$$

then 


$$
\begin{aligned}
& \frac{d \mathfrak{r}_{1}}{d s}=\mathfrak{q}^{2}\left|\begin{array}{lcc}
z_{1}-\left(S-\frac{k_{20}}{4 ! Q_{0}} S^{4}+\cdots\right) & 1-\frac{k_{20}}{3 ! Q_{0}} S^{3}+\cdots & -\frac{k_{20}}{Q_{0}} S+\cdots \\
z_{2}-\left(\frac{1}{2} S^{2}-\frac{k_{10}}{4 ! Q_{0}} S^{4}+\cdots\right) & S-\frac{k_{10}}{3 ! Q_{0}} S^{3}+\cdots & -\frac{k_{10}}{Q_{0}} S+\cdots \\
z_{3}-\left(\frac{1}{3 ! Q_{0}} S^{3}-\frac{Q_{0}^{\prime}}{4 ! Q_{0}^{2}} S^{4}+\cdots\right), \frac{1}{2 Q_{0}} S^{2}-\frac{Q_{0}^{\prime}}{3 ! Q_{2}^{0}} S^{3}+\cdots, \frac{1}{Q_{0}}-\frac{Q_{0}^{\prime}}{Q_{0}^{2}} S+\cdots
\end{array}\right| \\
& +2 \mathfrak{q} \frac{d \mathfrak{q}}{d s}\left|\begin{array}{lll}
z_{1}-\left(S-\frac{k_{20}}{4 ! Q_{0}} S^{4}+\cdots\right) & 1-\frac{k_{20}}{3 ! Q_{0}} S^{3}+\cdots & -\frac{h_{20}}{2 Q_{0}} S^{2}+\cdots \\
z_{2}-\left(\frac{1}{2 !} S^{2}-\frac{k_{10}}{4 ! Q_{0}} S^{4}+\cdots\right) & S-\frac{k_{10}}{3 ! Q_{0}} S^{3}+\cdots & 1-\frac{k_{10}}{2 Q_{0}} S^{2}+\cdots \\
z_{3}-\left(\frac{1}{3 ! Q_{0}} S^{3}-\frac{Q_{0}^{\prime}}{4 ! Q_{0}^{2}} S^{4}+\cdots\right), \frac{1}{2 Q_{0}} S^{2}-\frac{Q_{0}^{\prime}}{3 ! Q_{0}} S^{3}+\cdots, & \frac{1}{Q_{0}} S-\frac{Q_{0}^{\prime}}{2 Q_{0}^{2}} S^{2}+\cdots
\end{array}\right|,
\end{aligned}
$$

therefore

$$
\begin{aligned}
\lim _{s \rightarrow 0} \frac{d \mathfrak{r}_{1}}{d S} & =\mathfrak{q}^{2}\left|\begin{array}{ccc}
z_{1} & 1 & 0 \\
z_{2} & 0 & 0 \\
z_{3} & 0 & \frac{1}{Q_{0}}
\end{array}\right|+2 \mathfrak{q} \lim _{s \rightarrow 0} \frac{d \mathfrak{q}}{d S}\left|\begin{array}{ccc}
z_{1} & 1 & 0 \\
z_{2} & 0 & 1 \\
z_{3} & 0 & 0
\end{array}\right| \\
& =-\mathfrak{q}^{2} \frac{z_{2}}{Q_{0}}+2 \mathfrak{q} z_{3} \lim _{s \rightarrow 0} \frac{d \mathfrak{q}}{d S} .
\end{aligned}
$$

However we have

$$
\frac{d q}{d S}=\frac{\partial q}{\partial u^{i}} \frac{d u^{i}}{d S} \cdot \quad(i=1,2)
$$

If the affine normal at $Q$ passing through the origin, then we have

$$
\lim _{s \rightarrow 0} \frac{\partial q}{\partial u^{i}}=0 \cdot\left({ }^{15}\right) \quad(i=1,2)
$$

The values of $\lim _{s \in 0} \frac{d u^{1}}{d S}$ and $\lim _{s \rightarrow 0} \frac{d u^{2}}{d S}$ are different from zero in general. Thus we get

Theorem 2. The r-affine perpendicular distance from a point $z$ to the osculating plane at a point $x$ on $C$, has a stationary value, when zlies on

(15) W. Blaschke, loc. cit.; p. 111. 
the r-affine rectifying plane and the affine surface normal at the corresponding point on $E$ passes through the origin.

Cor. The affine perpendicular distance $\mathfrak{p}_{1}$ has a stationary value, when $z$ lies on the affine rectifying plane.

By the same method we have

$$
\lim _{s \rightarrow 0} \frac{d \mathfrak{x}_{2}}{d S}=\mathfrak{q}^{6}\left(\frac{z_{1}}{Q_{0}}+\frac{Q_{0}^{\prime}}{Q_{0}^{2}} z_{2}-\frac{k_{10}}{Q_{0}} z_{3}\right)-\frac{z_{2}}{Q_{0}} 6 \mathfrak{q}^{5} \lim _{s \rightarrow c} \frac{d \mathfrak{q}}{d S}
$$

Consequently we get the following

Theorem 3. The r-affine perpendicular distance from a point $z$ to the $r$-affine rectifying plane at a point $x$ on $C$, has a stationary value, when z lies on the r-affine principal normal, the affine surface normal at the corresponding point on $E$ passes through the origin and $Q_{0}{ }^{\prime}=0$.

Cor. The affine perpendicular distance $p_{2}$ has a stationary value, when z lies on the affine principal normal.

\section{$\$ 4$. Indicatrices of tangents, r-curvatures and $r$-covariant curvatures.}

The curves described by the end points of the vectors, from the origin, $\xi_{1},-\xi_{2}$ and $\xi_{3}+k_{1} \xi_{1}$, at a point $P$ on a curve $C(x)$ are called respectively the indicatrix of $r$-tangents, the indicatrix of $r$-principal normals and the indicatrix of covarant r-curvatures in affine space. We will here discuss the relations between a curve $C(x)$ and its indicatrices.

Denote by $\mathfrak{y}_{1}$ the coordinates of a point on the indicatrix $C_{1}$ of $r$ tangent and denote by $S_{1}$ the arc length of $C_{1}$, then we have

$$
\begin{aligned}
d S_{1} & =\mathfrak{q}_{1}\left|\mathfrak{y}_{1}^{\prime}, \mathfrak{y}_{1}^{\prime \prime}, \mathfrak{y}_{1}^{\prime \prime \prime}\right|^{\frac{1}{6}} d S \\
& =\mathfrak{q}_{1}\left|\xi_{2}, \frac{\xi_{3}}{Q} \cdot \frac{1}{Q}\left(-k_{1} \xi_{2}-k_{2} \xi_{1}\right)\right|^{\frac{1}{6}} d S,
\end{aligned}
$$

therefore

$$
d S_{1}=\frac{q_{1}}{q^{2}}\left(-k_{2}\right)^{\frac{1}{6}} d S
$$

Consequently $k_{2}$ is given by

$$
k_{2}=-\left(\frac{q d \bar{S}_{1}}{d \bar{S}}\right)^{6}
$$


Therefore by means of (2) and (8) in $\$ 1$, a geometrical meaning of $k_{2}$ is given:

Theorem 4. If we denote by $V_{2}^{\prime}$ the corresponding quantity on $C_{1}$ to $V_{2}$ on $C$ and denote by $V_{i}^{0}$ the corresponding quantities of the asymptotic curve on the unit surface to $V_{i}$ on $C$, then we have

$$
k_{2}=-\lim _{\Delta t \rightarrow 0} \frac{\sqrt{6} V_{1}^{1} V_{1}^{0}}{V_{2} \sqrt{V_{2}^{0}}} .
$$

Denote the point coordinates on the indicatrix $C_{2}$ of $r$-principal normals by $\mathfrak{y}_{2}$ and its arc length by $s_{2}$, then we have

$$
\begin{aligned}
d S_{2} & =\mathfrak{q}_{2}\left|\mathfrak{y}_{2}^{\prime}, \mathfrak{l}_{2}^{\prime \prime}, \mathfrak{y}_{2}^{\prime \prime \prime}\right|^{\frac{1}{6}} d S \\
& =\mathfrak{q}_{2}\left|\mathfrak{x}^{\prime \prime \prime}, \mathfrak{r}^{\mathrm{IV}}, \mathfrak{x}^{\mathrm{V}}\right|^{\frac{1}{6}} d S \\
& =\frac{\mathfrak{q}_{2}}{\sqrt{Q}}\left|\xi_{3}, k_{1} \xi_{2}+k_{2} \xi_{1},\left(k_{1}^{\prime}+k_{2}+\frac{2 Q^{\prime}}{Q} k_{1}\right) \xi_{2}+\left(k_{2}^{\prime}+\frac{2 Q^{\prime}}{Q} k_{2}\right) \xi_{1}\right|^{\frac{1}{6}} d S,
\end{aligned}
$$

consequently

$$
d S_{2}=\frac{\mathfrak{q}_{2}}{\mathfrak{q}^{3}}\left(k_{2}^{2}+k_{1}^{\prime} k_{2}-k_{1} k_{2}^{\prime}\right)^{\frac{1}{6}} d S .
$$

If we denote the point coordinates on the indicatrix of covariant $r$ curvatures by $\mathfrak{y}_{3}$ and its $r$-arc length by $S_{3}$; then we have

$$
d S_{3}=\mathfrak{q}_{3}\left|\mathfrak{y}_{3}^{\prime}, \mathfrak{y}_{3}^{\prime \prime} ; \mathfrak{y}_{3}^{\prime \prime \prime}\right|^{\frac{1}{6}} d S,
$$

now

$$
\begin{aligned}
\mathfrak{y}_{3} & =\xi_{3}+k_{1} \xi_{1}, \\
\mathfrak{y}_{3}^{\prime} & =-\left(k_{1} \xi_{2}+k_{2} \xi_{1}\right)+k_{1} \xi_{1}^{\prime}+k_{1}^{\prime} \xi_{1}=\left(k_{1}^{\prime}-k_{2}\right) \xi_{1}, \\
\mathfrak{y}_{3}^{\prime \prime} & =\left(k_{1}^{\prime}-k_{2}\right) \xi_{2}+\left(k_{1}^{\prime \prime}-k_{2}^{\prime}\right) \xi_{1}, \\
\mathfrak{y}_{3}^{\prime \prime \prime} & =\frac{1}{Q}\left(k_{1}^{\prime}-k_{2}\right) \xi_{3}+2\left(k_{1}^{\prime \prime}-k_{2}^{\prime}\right) \xi_{2}+\left(k_{1}^{\prime \prime \prime}-k_{2}^{\prime \prime}\right) \xi_{1} .
\end{aligned}
$$

Substituting (39) in (38) we get

$$
\begin{aligned}
d S_{3} & =\mathfrak{q}_{3}\left|\left(k_{1}^{\prime}-k_{2}\right) \xi_{1},\left(k_{1}^{\prime}-k_{2}\right) \xi_{2},\left(k_{1}^{\prime}-k_{2}\right) \frac{\xi_{3}}{Q}\right| \frac{1}{6} d S \\
& =\frac{\mathfrak{q}_{3}}{\mathfrak{q}}\left(k_{1}-k_{2}\right)^{\frac{1}{2}} d S .
\end{aligned}
$$

From this fact we get the following 
Theorem 5. If the straight line parallel to the vector $\xi_{3}+k_{1} \xi_{1}$ and passing through the point $x$ on a space curve, passes through a fixed point, then we have $\bar{S}_{3}=a+b \bar{S}$, where $a$ and $b$ are constants, and the two curves $C$ and $C_{3}$ are parallel curves.

Proof. Denote the fixed point by $z$, then we have

$$
\begin{gathered}
\gamma=x+\lambda\left(\xi_{3}+k_{1} \xi_{1}\right), \\
\xi^{\prime}=\left(1-\lambda k_{2}+\lambda k_{1}^{\prime}+\lambda^{\prime} k_{1}\right) \xi_{1}+\lambda^{\prime} \xi_{3}=0,
\end{gathered}
$$

consequently

$$
\lambda^{\prime}=0 \quad \text { or } \quad \lambda=\text { constant }
$$

and

$$
k_{2}-k_{1}^{\prime}=\frac{1}{\lambda}=\text { constant }
$$

Thus from (40) the result follows.

\section{$\S 5$. A geometrical meaning of the r-affine principal normal.}

Denote the coordinates of a point on the tangent surface of a space curve $C(x)$ by $\mathfrak{X}=\left\{X_{1}, X_{2}, X_{3}\right\}$, then we have

$$
\mathfrak{X}=\mathfrak{x}+\tau \mathfrak{l}^{\prime},
$$

where $\tau$ is the parameter and $x^{\prime}=\frac{d x}{d S}$.

By using the formulae (19).

$$
\begin{aligned}
X_{1}=x_{1}+\tau x_{1}^{\prime}= & S-\frac{k_{20}}{4 ! Q_{0}} S^{4}+\cdots+\tau\left(1-\frac{k_{20}}{3 ! Q_{0}} S^{3}+\cdots\right), \\
X_{2}=x_{2}+\tau x_{2}^{\prime}= & \frac{1}{2} S^{2}-\frac{k_{10}}{4 ! Q_{0}} S^{4}+\cdots+\tau\left(S-\frac{k_{10}}{3 ! Q_{0}} S^{3}+\cdots\right), \\
X_{3}=x_{3}+\tau x_{3}^{\prime}= & \frac{1}{3 ! Q_{0}} S^{3}-\frac{Q_{0}^{\prime}}{4 ! Q_{0}} S^{4}+\frac{A_{0}}{5 ! Q_{0}^{3}} S^{5}+\cdots \\
& +\tau\left(\frac{1}{2 Q_{0}} S^{2}-\frac{Q_{0}^{\prime}}{3 ! Q_{0}^{2}} S^{3}+\frac{A_{0}}{4 ! Q_{0}^{3}} S^{4}+\cdots\right)
\end{aligned}
$$

where $A_{0} \equiv 2 Q_{0}{ }^{\prime}-Q_{0} Q_{0}{ }^{\prime \prime}-Q_{0} k_{10}$.

As the point $\mathfrak{X}$ lies on the $r$-osculating plane,

$$
X_{3}=0,
$$


and we have

$$
\begin{aligned}
\text { (42) } \tau & =-\frac{\frac{1}{3 ! Q_{0}} S^{3}-\frac{Q_{0}^{\prime}}{4 ! Q_{0}^{2}} S^{4}+\frac{A_{0}}{5 ! Q_{0}^{3}} S^{5}+\cdots}{\frac{1}{2 Q_{0}} S^{2}-\frac{Q_{0}^{\prime}}{3 ! Q_{0}^{2}} S^{3}+\frac{A_{0}}{4 ! Q_{0}^{3}} S^{4}+\cdots} \\
& =-\frac{S}{3}\left(1-\frac{Q_{0}^{\prime}}{4 Q_{0}} S+\frac{A_{0}}{20 Q_{0}^{2}} S^{2}+\cdots\right)\left(1-\frac{Q_{0}^{\prime}}{3 Q_{0}} S+\frac{A_{0}}{12 Q_{0}} S^{2}+\cdots\right)^{-1} \\
& =-\frac{S}{3}\left(1-\frac{Q_{0}^{\prime}}{4 Q_{0}} S+\frac{A_{0}}{20 Q_{0}^{2}} S^{2}+\cdots\right)\left(1+\frac{Q_{0}^{\prime}}{3 Q_{0}} S-\frac{A_{0}}{12 Q_{0}^{2}} S^{2}+\frac{Q_{0}^{2}}{9 Q_{0}^{2}} S^{2}+\cdots\right) \\
& =-\frac{S}{3}\left(1+\frac{Q_{0}^{\prime}}{12 Q_{0}} S+\frac{5 Q_{0}^{2}-6 A_{0}}{180 Q_{0}^{2}} S^{2}+\cdots\right) \cdot
\end{aligned}
$$

Substituting (42) in $X_{1}$ and $X_{2}$ of (41) we have

$$
\begin{aligned}
X_{1}= & S-\frac{k_{20}}{4 ! Q_{0}} S^{4}+\cdots-\frac{S}{3}\left(1+\frac{Q_{0}^{\prime}}{12 Q_{0}} S+\frac{5 Q_{0}^{\prime 2}-6 A_{0}}{180 Q_{0}^{2}} S^{2}+\cdots\right)\left(1-\frac{k_{20}}{3 ! Q_{0}} S^{3}+\cdots\right) \\
= & \frac{2}{3} S-\frac{Q_{0}^{\prime}}{36 Q_{0}} S^{2}-\frac{5 Q_{0}^{\prime 2}-6 A_{0}}{540 Q_{\cup}^{2}} S^{3}+\cdots, \\
X_{2}= & \frac{1}{2} S^{2}-\frac{k_{10}}{4 ! Q_{0}} S^{4}+\cdots-\frac{S}{3}\left(1+\frac{Q_{0}^{\prime}}{12 Q_{0}} S+\frac{5 Q_{0}^{\prime 2}-6 A_{0}}{180 Q_{0}^{2}} S^{2}+\cdots\right) \\
& \quad\left(S-\frac{k_{10}}{3 ! Q_{0}} S^{3}+\cdots\right)=\frac{1}{6} S^{2}-\frac{Q_{0}^{\prime}}{36 Q_{0}} S^{3}+\cdots,
\end{aligned}
$$

and

$$
\left(\frac{d X_{1}}{d S}\right)_{s=0}=\frac{2}{3},\left(\frac{d^{2} X_{1}}{d S^{2}}\right)_{s=0}=-\frac{Q_{0}^{\prime}}{18 Q_{0}},\left(\frac{d^{3} X_{1}}{d S^{3}}\right)_{s=0}=-\frac{5 Q_{0}^{\prime 2}-6 A_{0}}{90 Q_{0}^{2}},
$$

$$
\left(\frac{d X_{2}}{d S}\right)_{s=0}=0,\left(\frac{d^{2} X_{2}}{d S^{2}}\right)_{s=0}=\frac{1}{3},\left(\frac{d^{3} X_{2}}{d S^{3}}\right)_{s=0}=-\frac{Q_{0}^{\prime}}{6 Q_{0}}
$$

therefore

$$
\left(\left|\begin{array}{cc}
X_{1}^{\prime} & X_{1}^{\prime \prime} \\
X_{2}^{\prime} & X_{2}^{\prime \prime}
\end{array}\right|\right)_{s=0}=\frac{2}{9}, \quad\left(\left|\begin{array}{cc}
X_{1}^{\prime} & X_{2}^{\prime \prime \prime} \\
X_{2}^{\prime} & X_{2}^{\prime \prime \prime}
\end{array}\right|\right)_{s=0}=-\frac{Q_{0}^{\prime}}{9 Q_{0}}
$$

Denote by $S^{*}$ the $r$-affine arc length of the curve $C^{*}$, which is obtained as the osculating plane section of the tangent surface of $C$, then we have 


$$
\left(\frac{d S^{*}}{d S}\right)_{s=0}=\left(\mathfrak{q}^{*}\left|\begin{array}{ll}
X_{1}^{\prime} & X_{1}^{\prime \prime} \\
X_{2}^{\prime} & X_{2}^{\prime \prime}
\end{array}\right|^{\frac{1}{3}}\right)_{s=0}=q^{*}\left(\frac{2}{9}\right)^{\frac{1}{3}}
$$

and

$$
\begin{aligned}
& \frac{d^{2} X_{1}}{d S^{* 2}}=\frac{d}{d S^{*}}\left(\frac{d X_{1}}{d S} \frac{d S}{d S^{*}}\right)=\frac{d}{d S^{*}}\left(\frac{1}{q^{*}} X_{1}^{\prime}\left|\begin{array}{cc}
X_{1}^{\prime} & X_{1}^{\prime \prime} \\
X_{2}^{\prime} & X_{2}^{\prime \prime}
\end{array}\right|^{-\frac{1}{3}}\right) \\
& =-\frac{\mathfrak{q}^{* \prime}}{\mathfrak{q}^{* 3}} X_{1}^{\prime}\left|\begin{array}{ll}
X_{1}^{\prime} & X_{1}^{\prime \prime} \\
X_{2}^{\prime} & X_{2}^{\prime \prime}
\end{array}\right|^{-\frac{2}{3}}+\frac{X_{1}^{\prime \prime}}{\mathfrak{q}^{* 2}}\left|\begin{array}{ll}
X_{1}^{\prime} & X_{1}^{\prime \prime} \\
X_{2}^{\prime} & X_{2}^{\prime \prime}
\end{array}\right|^{-\frac{2}{3}} \\
& -\frac{1}{3} \frac{X_{1}^{\prime}}{q^{* 2}}\left|\begin{array}{lll:ll}
X_{1}^{\prime} & X_{1}^{\prime \prime} & -\frac{5}{3} & X_{1}^{\prime} & X_{1}^{\prime \prime \prime} \\
X_{2}^{\prime} & X_{2}^{\prime \prime} & X_{2}^{\prime} & X_{2}^{\prime \prime \prime}
\end{array}\right|,
\end{aligned}
$$

then

$$
\begin{aligned}
\left(\frac{d^{2} X_{1}}{d S^{* 2}}\right)_{s * 0}= & -\frac{q^{* \prime}}{q^{* 3}} \frac{2}{3}\left(\frac{9}{2}\right)^{\frac{2}{3}}+\frac{1}{q^{* 2}}\left(-\frac{Q_{0}^{\prime}}{18 Q_{0}}\right)\left(\frac{9}{2}\right)^{\frac{2}{3}} \\
& \quad-\frac{1}{3 q^{* 2}}\left(\frac{2}{3}\right)\left(\frac{9}{2}\right)^{\frac{5}{3}}\left(-\frac{Q_{0}^{\prime}}{9 Q_{0}}\right) \\
= & -\frac{2}{3}\left(\frac{9}{2}\right)^{\frac{2}{3}} \frac{q^{* \prime}}{\mathfrak{q}^{* 3}}+\frac{1}{18 \mathfrak{q}^{* 2}}\left(\frac{9}{2}\right)^{\frac{2}{3}} \frac{Q_{0}^{\prime}}{Q_{0}} \\
= & \frac{1}{18 \mathfrak{q}^{* 2}}\left(\frac{9}{2}\right)^{\frac{2}{3}}\left(\frac{Q_{0}^{\prime}}{Q_{0}}-12 \frac{\mathfrak{q}^{* \prime}}{\mathfrak{q}^{*}}\right) \\
= & \frac{1}{3 \mathfrak{q}^{* 2}}\left(\frac{9}{2}\right)^{\frac{2}{3}}\left(\frac{\mathfrak{q}_{0}^{\prime}}{\mathfrak{q}_{0}}-\frac{2 \mathfrak{q}^{* \prime}}{\mathfrak{q}^{*}}\right) .
\end{aligned}
$$

Hence if $q=c q^{* 2}$ where $c$ is a constant of integration, we have

$$
\left(\frac{d^{2} X_{1}}{d S^{* 2}}\right)_{s=0}=0
$$

Consequently in this case the $r$-affine principal normal of $C$ coincides with the $r$-affine normal of $C^{*}$. Thus we have an extension of $R$. Weitzenböck's theorem : $\left({ }^{16}\right)$

Theorem 6. For the plane curve $C^{*}$ determined by the plane section of the tangent surface of a space curve $C$ with the osculating plane at a

(16) R. Weitzenböck, Wiener Sitzungsberichte, 127 (1918), pp. 969-997. 
point on $C$, if we take as the unit curve the plane section of the unit surface with the plane, which is parallel to the osculating plane at r., passes through the corresponding point $\mathrm{e}$ on $E$, and the origin is taken as $\mathrm{q}^{*}=c_{\sqrt{\mathrm{q}}}$, the $r$-affine principal normal of $C$ coincides with the $r$-affine normal of $C^{*}$.

\section{§. Natural equations.}

Let $C$ and $C^{*}$ be two curves defined in terms of their respective $r$-affine arc length $S$, and let point upon each with the same values of $S$ correspond to each other. Let us assume, furthermore, that at corresponding points the $r$-affine curvature and the $r$-affine torsion have the same value. We will prove that $C$ and $C^{*}$ are transformable by affine transformation: From the assumption $\mathfrak{l}_{\text {and }} \mathrm{x}^{*}$ must satisfy the differential equation (16).

$$
\xi_{3}^{\prime}+k_{1} \xi_{2}+k_{2} \xi_{1}=0
$$

or

$$
\left(Q r^{\prime \prime \prime}\right)^{\prime}+k_{1} \mathfrak{x}^{\prime \prime}+k_{2} x^{\prime}=0
$$

At first we consider the case where both $r$-rectifying planes at the corresponding points $x$ and $\mathfrak{r}^{*}$ are parallel. In this case $x_{i}^{\prime}(i=1,2,3)$ must be a system of solutions of the linear differential equation of the third order.

$$
\left(Q y^{\prime \prime}\right)^{\prime}+k_{1} y^{\prime}+k_{2} y=0
$$

Take $y_{i}(i=1,2,3)$ as the linearly independent solutions of $(46)$, then we have

$$
\left|\begin{array}{lll}
\left(Q y_{1}^{\prime \prime}\right)^{\prime} & y_{1}^{\prime} & y_{1} \\
\left(Q y_{2}^{\prime \prime}\right)^{\prime} & y_{2}^{\prime} & y_{2} \\
\left(Q y_{3}^{\prime \prime}\right)^{\prime} & y_{3}^{\prime} & y_{3}
\end{array}\right|=0
$$

therefore

$$
\left|\begin{array}{lll}
y_{1}^{\prime \prime} & y_{1}^{\prime} & y_{1} \\
y_{2}^{\prime \prime} & y_{2}^{\prime} & y_{3} \\
y_{3}^{\prime \prime} & y_{3}^{\prime} & y_{2}
\end{array}\right|=\frac{C}{Q},
$$

where $c$ is a constant of integration.

By multiplication of a suitable constant we can make the above constant equal to one so that 


$$
\left|\begin{array}{lll}
y_{1}^{\prime \prime} & y_{1}^{\prime} & y_{1} \\
y_{2}^{\prime \prime} & y_{2}^{\prime} & y_{2} \\
y_{3}^{\prime \prime} & y_{3}^{\prime} & y_{3}
\end{array}\right|=\frac{1}{Q} .
$$

Now let us put

$$
x_{i}^{\prime}=a_{i 1} y_{1}+a_{i 2} y_{2}+a_{i 3} y_{3}, \quad(i=1,2,3)
$$

then we have

$$
\left|r^{\prime}, \underline{x}^{\prime \prime}, \underline{x}^{\prime \prime \prime}\right|=a\left|y, y^{\prime}, y^{\prime \prime}\right|=\frac{a}{Q},
$$

where $\quad a \equiv\left|a_{i k}\right| \neq 0$.

Let us take $a=1$ and put

$$
x_{i}^{*}=\int_{0}^{s} y_{i} d S, \quad(i=1,2,3)
$$

thus the general solutions of (45) are given by the following equations

$$
x_{i}=a_{i 0}+a_{i 1} x_{1}^{*}+a_{i 2} x_{2}^{*}+a_{i 3} x_{3}^{*}, \quad(i=1,2,3)
$$

where $\left|\mathfrak{x}^{\prime}, r^{\prime \prime}, \underline{x}^{\prime \prime \prime}\right|=\frac{1}{\mathfrak{q}^{6}}=\frac{1}{Q}$.

In case where both $r$-rectifying planes at the corresponding points $\mathfrak{x}$ and $\mathfrak{x}^{*}$ are not parallel, if $x^{*}$ be affinely equivalent to $\mathfrak{q}$, the surface must be a quadric or an affine transform of a periodic surface. $\left({ }^{17}\right)$ In this case we can make the corresponding $r$-rectifying planes parallel by an equivalent affine transformation, consequently it is reduced to the preceding case. Hence we have the following.

Theorem 7. If the r-affine curvature $k_{1}$ and the r-affine torsion $k_{2}$ are given as analytic functions of the r-affine arc length $S$, a curve which has $k_{1}$ as the r-affine curvature and $k_{2}$ as the r-affine torsion, is uniquely determined except for the translation in general. In case where the unit surface is a periodic surface such that $q^{*}=\mathfrak{q}$, the curve is uniquely determined except for translation and a rotation, and in case where the unit surface is a quadric, the curve is uniquely determined except for the equivalent affine transformations and translation.

We call $k_{1}=k_{1}(S)$ and $k_{2}=k_{2}(S)$ the natural equations.

(17) J. Hirakawa, E. R. G. II, loc. cit., Theorem 1 in 81 , p. 86. 


\section{$\S 7$. Special Curves.}

\section{Curves of constant r-curvature.}

The centre of r-curvature is defind as follows:

$$
y=\mathrm{r}+\frac{1}{k_{1}} \mathrm{r}^{\prime \prime} .
$$

Denote by $\mathfrak{r}_{2}$ the $r$-affine perpendicular distance from the centre of $r$-curvature to the $r$-rectifying plane at a point $x$ on a space curve $C$, then we have

$$
\mathfrak{x}_{2}=\left|-\frac{1}{k_{1}} \mathrm{r}^{\prime \prime}, \xi_{1}, \xi_{3}\right|=\frac{1}{k_{1}} .
$$

If the relative curvature is constant, then we have

$$
\mathfrak{y}^{\prime}=\mathfrak{x}^{\prime}+\frac{1}{k_{1}} \mathrm{r}^{\prime \prime \prime \prime} .
$$

Thus we know the tangent at $y$ on the curve described by the centre of r-curvature is parallel to the r-rectifying plane at $\mathrm{x}$.

\section{Curves of constant torsion.}

The following theorem is an extension of that given by $E$. Cech:

Theorem 8. A necessary and sufficient condition that the r-affine principal normals of a space curve $C(\mathrm{x})$ be parallel to a fixed plane is that $k_{2}$ is identically zero.

Proof. From (16) we have

$$
\xi_{3}^{\prime}+k_{1} \xi^{\prime \prime}=0 .
$$

Denote by a the normal unit vector of a plane, which satisfies the following relations

$$
\left(\mathfrak{a}_{c}^{\prime \prime}\right)=\left(a_{c}^{\prime \prime \prime \prime}\right)=\left(a \xi_{3}\right)=0,
$$

then by means of (i) and (ii) we have

$$
\left(a \xi_{3}^{\prime}\right)=0 \text {, }
$$

and consequently

$$
\left(a^{\prime} \underline{c}^{\prime \prime}\right)=\left(a^{\prime} \xi_{3}\right)=0 .
$$


From (ii) and (iv) we get

$$
\mathfrak{a}^{\prime}=\lambda \mathfrak{a}
$$

by virtue of

$$
\xi^{\prime \prime}+\sigma \xi_{3} \neq 0
$$

However from $a^{2}=1$, we have $a a^{\prime}=0$, from which follows

$$
\lambda=0
$$

and consequently

$$
\mathfrak{a}^{\prime}=0, \text { or } a=a \text { constant vector. }
$$

Hence the $r$-affine principal normals are always parallel to a fixed plane.

Conversely, if the $r$-affine principal normals are always parallel to a fixed plane, it is easily seen that the $r$-affine torsion is constant.

\section{R-affine Bertrand curves.}

The curves such that the $r$-affine principal normals of a curve are the $r$-affine principal normals of another curve, are called r-affine Bertrand curves. We will seek for a necessary and sufficient condition that the two curves $C(x)$ and $C^{*}\left(\mathfrak{x}^{*}\right)$ belong to the same Bertrand curves. By assumption the following relations hold:

$$
\begin{aligned}
& r^{*}=x+\lambda r^{\prime \prime}, \\
& r^{* \prime \prime}=\alpha r^{\prime \prime}
\end{aligned}
$$

Put

$$
\frac{d S^{*}}{d S}=\varphi
$$

then we have

$$
\begin{aligned}
& \mathcal{H}^{* \prime} \varphi=r^{\prime}+\lambda^{\prime} \hat{c}^{\prime \prime}+\lambda r^{\prime \prime \prime},
\end{aligned}
$$

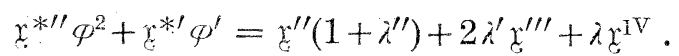

From (16) we obtain

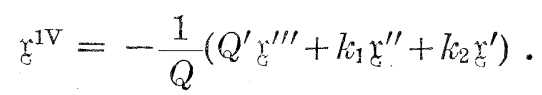


Substituting (57) in (56) we have

$$
\mathfrak{r}^{* \prime \prime} \phi^{2}+r^{* \prime} \phi^{\prime}=-\frac{\lambda k_{2}}{Q} \mathfrak{x}^{\prime}+\left(1+\lambda^{\prime \prime}-\frac{\lambda k_{1}}{Q}\right) \mathfrak{r}^{\prime \prime}+\left(2 \lambda^{\prime}-\frac{\lambda Q^{\prime}}{Q}\right) \mathfrak{x}^{\prime \prime \prime},
$$

substituting (54) and (55)

$$
\begin{aligned}
\alpha \mathcal{P}^{2} \mathrm{x}^{\prime \prime}+\frac{\phi^{\prime}}{\varphi}\left(\mathrm{x}^{\prime}+\lambda^{\prime} \mathrm{x}^{\prime \prime}+\lambda \mathrm{x}^{\prime \prime \prime}\right) & \\
= & -\frac{\lambda k_{2}}{Q} \mathrm{x}^{\prime}+\left(1+\lambda^{\prime \prime}-\frac{\lambda k_{1}}{Q}\right) \mathrm{x}^{\prime \prime}+\left(2 \lambda^{\prime}-\frac{\lambda Q^{\prime}}{Q}\right) \mathrm{x}^{\prime \prime \prime} .
\end{aligned}
$$

Consequently from the fact that $\mathfrak{x}^{\prime}, \mathfrak{x}^{\prime \prime}$ and $\mathfrak{x}^{\prime \prime \prime}$ are linearly indpendent we have

$$
\begin{gathered}
\frac{\phi^{\prime}}{\phi}+\frac{\lambda k_{2}}{Q}=0, \\
\alpha \phi^{2}+\frac{\lambda^{\prime} \phi^{\prime}}{\varphi}=1+\lambda^{\prime \prime}-\frac{\lambda k_{1}}{Q}, \\
\frac{\lambda \phi^{\prime}}{\varphi}=2 \lambda^{\prime}-\frac{\lambda Q^{\prime}}{Q} .
\end{gathered}
$$

From (62) we have

$$
\varphi=C \frac{\lambda^{2}}{Q}
$$

Differentiating (54) we have

$$
\hat{\gamma}^{* \prime \prime \prime} \phi=\alpha^{\prime} \underline{x}^{\prime \prime}+\alpha \chi^{\prime \prime \prime \prime},
$$

then

$$
\left|\phi r^{* \prime}, x^{* \prime \prime}, \phi r^{* \prime \prime \prime}\right|=\frac{\phi^{2}}{Q^{*}},
$$

therefore by means of (54), (55) and (64)

$$
\frac{\phi^{2}}{Q^{*}}=\left|r^{\prime}+\lambda^{\prime} \underline{g}^{\prime \prime}+\lambda \gamma^{\prime \prime \prime}, a \gamma^{\prime \prime}, \alpha^{\prime} \chi^{\prime \prime}+\alpha \gamma^{\prime \prime \prime}\right|=\frac{\alpha^{2}}{Q},
$$

then

$$
\varphi= \pm \sqrt{\frac{Q^{*}}{Q}} \alpha .
$$


Eliminating $\phi^{\prime} / \varphi$ from (60) and (62)

$$
k_{2}=\frac{Q^{\prime}}{\lambda}-\frac{2 Q \lambda^{\prime}}{\lambda^{2}}=\lambda\left(\frac{Q}{\lambda^{2}}\right)^{\prime}=\lambda\left(\frac{C}{\phi}\right)^{\prime}=-\frac{C \lambda \mathcal{P}^{\prime}}{\phi^{2}} .
$$

Now we can put

$$
r=\mathfrak{c}^{*}+\lambda^{*} \mathfrak{r}^{* \prime \prime},
$$

then by means of (53) and (54) we have

$$
\lambda^{*}=-\frac{\lambda}{\alpha} .
$$

The following relation can be seen in the same way as (66) was obtained :

$$
\begin{aligned}
k_{2}^{*} & =\lambda^{*}\left(\frac{Q^{*}}{\lambda^{* 2}}\right)^{\prime}=C^{*} \lambda^{*} \frac{\phi^{\prime}}{\varphi}=-\frac{C^{*} \lambda}{\alpha \varphi} \phi^{\prime} \\
& =-C^{*}\left( \pm \frac{1}{\phi^{2}} \sqrt{\frac{\overline{Q^{*}}}{Q}} \lambda \phi^{\prime}\right)= \pm \frac{C^{*}}{C} \sqrt{\frac{Q^{*}}{Q}} k_{2} .
\end{aligned}
$$

However we have

$$
\lambda^{*}=-\frac{\lambda}{\alpha}=-\lambda\left( \pm \frac{1}{\phi} \sqrt{\frac{Q^{*}}{Q}}\right)=-\lambda\left( \pm \frac{Q}{C \lambda^{2}} \sqrt{\frac{Q^{*}}{Q}}\right)=\mp \frac{\sqrt{Q Q^{*}}}{C \lambda},
$$

then

$$
\lambda \lambda^{*}=\mp \frac{\sqrt{Q_{Q^{*}}}}{C},
$$

therefore

$$
|C|=\left|C^{*}\right|
$$

Thus we have the conditions

$$
\frac{k_{2}^{*}}{\sqrt{Q^{*}}}= \pm \frac{k_{2}}{\sqrt{Q}}
$$

that is

$$
\frac{k_{2}^{*}}{q^{* 3}}= \pm \frac{k_{2}}{q^{3}}
$$

Hence we have following 
Theorem 9. A necessary and sufficient condition that two curves belong to the same Bertrand curves is that the relation

$$
\frac{k_{2}^{*}}{q^{* 3}}= \pm \frac{k_{2}}{q^{3}}
$$

holds.

Remarks. When $q=$ constant, this is reduced to the theorem given by Mr. S. Ogiwara. $\left({ }^{18}\right)$

\section{Chapter III. Theory of surfaces.}

\section{$\S 1$. Relations between two surfaces with their . parallel tangent planes.}

Let us make corresponce between a point $P(\mathfrak{x})$ on a surface $F(\mathfrak{c})$ and a point $Q(e)$ on a surface, $E(e)$ such that the tangent planes at $P$ and $Q$ are parallel to each other. Then we can put

$$
\mathrm{e}_{i}=-a_{i}^{\sigma} \mathfrak{\varepsilon}_{\sigma},
$$

where $i, \sigma=1,2$ and in euclidean $\operatorname{space}\left({ }^{19}\right)$ we have

$$
\begin{gathered}
a_{i}^{k}=-D_{\alpha_{\imath}} g^{\alpha_{k}}, \\
a \equiv a_{1}^{1} a_{2}^{2}-a_{1}^{2} a_{2}^{1}=\frac{D}{g}=K^{*}=\frac{\bar{K}(x)}{\bar{K}(\mathfrak{e})} .
\end{gathered}
$$

Now we will seek for another form of $a_{i}^{k}$ in affine space. Differentiate (1) with respect to $u^{k}$, then we have

$$
\begin{gathered}
\text { (4) } l_{i k}=\left|e_{1}, e_{2}, \frac{\partial^{2} \mathfrak{e}}{\partial u^{i} \partial u^{k}}\right|=-\left|a_{1}^{\sigma} \mathfrak{x}_{\sigma}, a_{2}^{\lambda} \mathfrak{\varkappa}_{\lambda}, a_{i}^{\mu} \frac{\partial^{2} x}{\partial u^{\mu} \partial u^{k}}\right|=-a \alpha_{i}^{\mu} L_{\mu k}, \\
l_{i k} L^{k j}=-a \alpha_{i}^{\mu} \delta_{\mu}^{j}=-a a_{i}^{j},
\end{gathered}
$$

then

$$
a_{i}^{j}=-\frac{1}{a} l_{i \alpha} L^{\alpha j}
$$

(18) Ueber affine Bertrandsche Kurven, Jap. Journ. of Math. IV (1927), pp. 33- 99.

(19) J. Hirakawa, E. R. G., IV, loc. cit., (61), (66) and (67) in 88, p. 117. 
therefore

$$
a \equiv a_{1}^{1} a_{2}^{2}-a_{1}^{2} a_{2}^{1}=\frac{l}{a^{2} L},
$$

where $l=l_{11} l_{22}-l_{12}^{2}, \quad L=L_{11} L_{22}-L_{12}^{2}$.

Consequently we have

$$
\frac{l}{L}=a^{3}=K^{* 3}
$$

Now let us denote the affine areal element by $d O$, then by means of $d O(\mathfrak{e})=|l|^{\frac{1}{4}} d u d v$ and $d O(\mathfrak{x})=|L|^{\frac{1}{4}} d u d v$, we obtain

$$
\frac{d O(e)}{d O(x)}=K^{* \frac{3}{4}}
$$

and

$$
\bar{K}^{\frac{3}{4}}(\mathfrak{e}) d O(e)=\bar{K}^{\frac{3}{4}} d O(\mathfrak{x})=d \omega,
$$

where $d \omega$ is the areal element on the unit sphere.

Consequently if the surface $F(x)$ be a convex closed surface, then we have

$$
\oint \bar{K}^{\frac{3}{4}}(x) d O(x)=\oint d \omega=4 \pi
$$

\section{$\S 2$. Fundamental quantities and fundamental equations.}

We take a convex closed surface $E(\mathrm{e})$ as the unit surface and we will discuss the relative geometric properties of the surface $F(\mathfrak{x})$ for the unit surface $E(\mathfrak{e})$.

Let us denote the affine perpendicular distance from the origin to the tangent plane at the point $Q(\mathrm{e})$ on $E(\mathrm{e})$ by $\mathfrak{q}$, and consider the following covariant vector

$$
\xi_{i k}=\mathfrak{q} \mathfrak{r}_{i k}-\mathfrak{q}_{i} \mathfrak{x}_{k}-\mathfrak{q}_{k} \mathfrak{x}_{i},
$$

where $\mathfrak{r}_{i k}$ denotes a covariant differentiation with respect to the fundamental tensor

$$
g_{i k}=\left(\overline{\mathfrak{X}} \frac{\partial^{\dot{2}} \mathfrak{x}}{\partial u^{i} \partial u^{k}}\right),
$$

where $\overline{\mathfrak{X}}=\frac{\mathfrak{x}_{1} \times \mathfrak{r}_{2}}{\left|L_{11} L_{22}-L_{12}^{2}\right|^{\frac{1}{4}}}$ and $L_{i k}=\left|\mathfrak{x}_{1}, \mathfrak{x}_{2}, \frac{\partial^{2} \mathrm{x}}{\partial u^{i} \partial u^{k}}\right|$. 
Let us put

$$
\mathfrak{X}=\frac{\mathfrak{x}_{1} \times x_{2}}{\mathfrak{q}|L|^{\frac{1}{4}}}
$$

then we have

$$
\begin{aligned}
g_{i k}=\mathfrak{x} \xi_{i k}=\frac{\left|\mathfrak{x}_{1}, x_{2}, \xi_{i k}\right|}{\mathfrak{q}|L|^{\frac{1}{4}}} & =\left|\frac{l}{L}\right| \frac{\frac{1}{4} \mid \frac{\left|x_{1}, x_{2}, \xi_{i k}\right|}{\left|\mathfrak{e}_{1}, \mathfrak{e}_{2},-\mathfrak{e}\right|}}{} \\
& =K^{* \frac{3}{4}} \frac{\left|x_{1}, \mathfrak{x}_{2}, \xi_{i k}\right|}{\left|\mathfrak{e}_{1}, \mathfrak{e}_{2},-\mathfrak{e}\right|}=g_{k i} .
\end{aligned}
$$

The $r$-affine normal vector is defined by the vector

$$
\mathfrak{l}=\frac{1}{2} g^{\alpha \beta} \xi_{\alpha \beta} \text {. }
$$

which is covariant for the affine transformation and the parameter transformation.

Then we have

$$
\mathfrak{y} \mathfrak{x}=1 \text {. }
$$

By covariant differentiation of (14) and (15) we get

$$
\begin{gathered}
\mathfrak{y}_{l}=\frac{1}{2} g^{\alpha \beta} \xi_{\alpha \beta, l}, \\
\mathfrak{y}_{l} \mathfrak{x}+\mathfrak{y} \mathfrak{x}_{l}=0 .
\end{gathered}
$$

Let us put

$$
A_{i k, l}=\mathfrak{X} \xi_{i k, l}, \quad(i, k, l=1,2)
$$

then we take $A_{i k, l}$ as the quantities of the third order.

By means of (16) and (17) we obtain

$$
\mathfrak{y}_{l} \mathfrak{X}=\frac{1}{2} g^{\alpha_{\beta}} \xi_{\alpha \beta, l} \mathfrak{X}=\frac{1}{2} g^{\alpha_{\beta}} A_{\alpha \beta, l}=-\mathfrak{y} \mathfrak{X}_{l} \equiv \frac{1}{2} V_{l} .
$$

From (13) by means of $g_{i k, l}=0$ we have

$$
A_{i k, l}=\mathfrak{X} \xi_{i k, l}=-\mathfrak{X}_{l} \xi_{i k}=A_{k i, l} .
$$


Now we prove that $V_{l}$ is equal to zero: Take the asymptotic curves as parametric curves, then we have

$$
\begin{aligned}
&\mathfrak{l})_{l}=g^{12} \xi_{12, l} \\
&=g^{12}\left(\frac{\partial \xi_{12}}{\partial u^{l}}-\Gamma_{1 l}^{\lambda} \xi_{\lambda 2}-\Gamma_{l 2}^{\lambda} \xi_{1 \lambda}\right) \\
&=g^{12}\left(\mathfrak{q} x_{12 l}+\mathfrak{q}_{l} x_{12}-\mathfrak{q}_{1} x_{2 l}-\mathfrak{q}_{2} x_{1 l}-\mathfrak{q}_{1 l} \mathfrak{x}_{2}-\mathfrak{q}_{2 l} \mathfrak{x}_{1}-\Gamma_{1 l}^{\lambda} \xi_{\lambda 2}-\Gamma_{l 2}^{\lambda} \xi_{1 \lambda}\right), \\
& \frac{1}{2} V_{l}=\mathfrak{x} \mathfrak{y}_{l}=g^{12}\left\{\frac{\left|x_{1}, \mathfrak{x}_{2}, \mathfrak{x}_{12 l}\right|}{\left|L_{12}\right|^{\frac{1}{2}}}+\mathfrak{q}_{l} g_{12}-\mathfrak{q}_{1} g_{2 l}-\mathfrak{q}_{2} g_{1 l}-\left(\Gamma_{1 l}^{1}+\Gamma_{l 2}^{2}\right) g_{12}\right\},
\end{aligned}
$$

where

$$
\begin{aligned}
& \Gamma_{1 l}^{1}=\Gamma_{1 l, \mu} g^{u 1}=\Gamma_{1 l, 2} g^{21}=\frac{1}{2}\left(\frac{\partial g_{12}}{\partial u^{l}}+\frac{\partial g_{l 2}}{\partial u^{1}}-\frac{\partial g_{1 l}}{\partial u^{2}}\right) g^{12}, \\
& \Gamma_{l 2}^{2}=\Gamma_{l 2, \mu} g^{\mu 2}=\Gamma_{l 2,1} g^{12}=\frac{1}{2}\left(\frac{\partial g_{l 1}}{\partial u^{2}}+\frac{\partial g_{21}}{\partial u^{l}}-\frac{\partial g_{l 2}}{\partial u^{1}}\right) g^{12},
\end{aligned}
$$

and

$$
\mathfrak{q}_{l} g_{12}-\mathfrak{q}_{1} g_{2 l}-\mathfrak{q}_{2} g_{1 l}=0
$$

Conseqüently we get

$$
\begin{aligned}
\frac{1}{2} V_{l}=\mathfrak{X} \mathfrak{y}_{l} & =g^{12}\left(\frac{\left|x_{1}, x_{2}, x_{12 l}\right|}{\left|L_{12}\right|^{\frac{1}{2}}}-\frac{\partial g_{12}}{\partial u^{l}} g^{12}\right) \\
& =g^{12}\left(\frac{1}{2\left|L_{12}\right|^{\frac{1}{2}}} \frac{\partial L_{12}}{\partial u^{l}}-\frac{1}{2} \frac{\partial L_{12}}{\partial u^{l}} \frac{1}{\left|L_{12}\right|^{\frac{1}{2}}}\right),
\end{aligned}
$$

therefore

$$
V_{l}=0
$$

that is

$$
g^{\alpha \beta} A_{\alpha \beta i}=0 . \quad(i=1,2)
$$

These equations are the conditions of apolarity.

Let us put

$$
\xi_{i k}=a_{i k \dot{c}_{\alpha}}^{\alpha}+b_{i k} \mathfrak{l},
$$

then by multiplication of $\mathfrak{X}$ and $-\mathfrak{X}_{l}$ we have

$$
b_{i k}=g_{i k} \text {, }
$$


and

$$
a_{i k}^{\alpha}=\mathfrak{q} A_{i k}^{\alpha} \equiv A_{i k}^{* \alpha} .
$$

Therefore we have

$$
\xi_{i k}=A_{i k}^{* \alpha} r_{\alpha}+g_{i k} \mathfrak{y}=\mathfrak{q} A_{i k}^{\alpha} \mathfrak{r}_{\alpha}+g_{i k} \mathfrak{y} .
$$

Let us put

$$
\mathfrak{y}_{i}=b_{i}^{\alpha} \mathfrak{c}_{\alpha}+C_{i} \mathfrak{y},
$$

then by means of $\mathfrak{X} \mathfrak{y}_{i}=\mathfrak{X} \mathfrak{x}_{\alpha}=0$ and $\mathfrak{X} \mathfrak{y}=1$ we have $C_{i}=0$.

Differentiating (25) covariantly with respect to $u^{k}$ and putting

$$
\mathfrak{X} \mathfrak{y}_{i k}=B_{i k} \equiv \frac{B_{i k}^{*}}{\mathfrak{q}},
$$

we have

$$
\mathfrak{y}_{i}=\mathfrak{q} B_{i}^{\alpha} \mathfrak{r}_{\alpha}=B_{i}^{* \alpha} \mathfrak{r}_{\alpha}
$$

where $B_{i}^{k}=B_{i \sigma} g^{\sigma k}$.

From (17) and (21) we have

$$
B_{i k}=\mathfrak{y}_{i k} \mathfrak{X}=-\mathfrak{y}_{i} \mathfrak{X}_{k}=\mathfrak{y} \mathfrak{X}_{k i}=B_{k i} .
$$

It is easily seen that the vectors $\mathfrak{X}, \mathfrak{X}_{1}$ and $\mathfrak{X}_{2}$ are linearly independent of the following relations

$$
\left|\mathfrak{X}, \mathfrak{X}_{1}, \mathfrak{X}_{2}\right|\left|\mathfrak{y}, \mathfrak{x}_{1}, \mathfrak{x}_{2}\right|=\left|\begin{array}{ccc}
1 & 0 & 0 \\
0 & -\frac{g_{11}}{q} & -\frac{g_{12}}{q} \\
0 & -\frac{g_{12}}{q} & -\frac{g_{22}}{q}
\end{array}\right|=\frac{g}{\mathfrak{q}^{2}},
$$

then

$$
\left|\mathfrak{X}, \mathfrak{X}_{1}, \mathfrak{X}_{2}\right|=\frac{g}{q^{3}|g|^{\frac{1}{2}}} .
$$

Consequently we can put

$$
\mathfrak{X}_{i k}=a_{i k}^{\alpha} \mathfrak{X}_{\alpha}+b_{i k} \ddot{X} .
$$


Let us form the scalar product of (31) by $\mathfrak{y}$ we have

$$
b_{i k}=B_{i k} \text {. }
$$

By the definition of $\mathfrak{X}$ we get

$$
\mathfrak{X} \mathfrak{x}_{l}=0,
$$

and differentiating covariantly

$$
\begin{aligned}
& \mathfrak{X}_{i} \mathfrak{x}_{l}=-\mathfrak{X} \mathfrak{x}_{l i}=-\frac{g_{l i}}{\mathfrak{q}}, \\
& \mathfrak{X}_{i k} \mathfrak{X}_{l}=\frac{\mathfrak{q}_{k}}{\mathfrak{q}^{2}} g_{l i}-\mathfrak{X}_{i} \mathfrak{x}_{l l k} .
\end{aligned}
$$

Hence from (20) we obtain

$$
\begin{aligned}
-\mathfrak{x}_{i} \mathfrak{x}_{l k} & =-\frac{\mathfrak{x}_{i}}{\mathfrak{q}}\left(\xi_{k l}+\mathfrak{q}_{l} \mathfrak{x}_{k}+\mathfrak{q}_{k} \mathfrak{x}_{l}\right) \\
& =\frac{1}{q}\left(A_{k l, i}+\frac{\mathfrak{q}_{l}}{\mathfrak{q}} g_{i k}+\frac{\mathfrak{q}_{k}}{\mathfrak{q}} g_{i l}\right),
\end{aligned}
$$

and substituting in (34)

$$
\mathfrak{x}_{i k} \Upsilon_{l}=\frac{1}{\mathfrak{q}}\left(A_{k l i}+\frac{\mathfrak{q}_{l}}{\mathfrak{q}} g_{i k}+\frac{2 \mathfrak{q}_{k}}{\mathfrak{q}} g_{i l}\right) .
$$

Consequently forming the scalar product of (31) by $x_{l}$, we have

$$
a_{i k}^{\alpha} g_{\alpha l}=-\left(A_{k l i}+\frac{\mathfrak{q}_{l}}{\mathfrak{q}} g_{i k}+\frac{2 q_{k}}{\mathfrak{q}} g_{i l}\right) \equiv-E_{i l k}
$$

Thus the equation (31) becomes

$$
\mathfrak{X}_{i k}=-E_{i k}^{\alpha} \mathfrak{X}_{\alpha}+B_{i k} \mathfrak{X}
$$

The equations (24), (27) and (38) are the fundamental equations.

\section{\$3. R-affine lines of curvature and $r$-affine principal curvatures.}

The $r$-affine line of curvature is such a curve that the $r$-affine surface normal along the curve describes a developable surface. 
From the definition, putting,

$$
\mathfrak{\jmath}=\mathfrak{x}+R \mathfrak{y},
$$

we have

$$
d \mathfrak{z}=d \mathfrak{x}+R d \mathfrak{y}+d R \mathfrak{l} \equiv=\sigma \mathfrak{y},
$$

Substituting (27)

$$
\begin{aligned}
& \mathfrak{x}_{i} d u^{i}+R B_{i}^{* \alpha} \mathfrak{x}_{\alpha} d u^{i}+(d R-\sigma) \mathfrak{y}=0, \\
& \left(\mathfrak{i}_{i}+R B_{i}^{* \alpha} \mathfrak{x}_{\alpha}\right) d u^{i}+(d R-\sigma) \mathfrak{y}=0 .
\end{aligned}
$$

From (40) eliminating $d u^{1}, d u^{2}$ and $d R-\sigma$ we have

$$
\left|\mathfrak{x}_{1}+R\left(B_{1}^{*} \mathfrak{x}_{1}+B_{1}^{* 2} \mathfrak{\Upsilon}_{2}\right), \mathfrak{x}_{2}+R\left(B_{2}^{* 1} \mathfrak{r}_{1}+B_{2}^{* 2} \mathfrak{c}_{2}\right), \mathfrak{y}\right|=0,
$$

then

$$
\begin{aligned}
\frac{1}{R^{2}}\left|\mathfrak{x}_{1}, \mathfrak{x}_{2}, \mathfrak{y}\right| & +\frac{1}{R}\left\{\left|\mathfrak{x}_{1}, B_{2}^{* 1} \mathfrak{x}_{1}+B_{2}^{* 2} \mathfrak{x}_{2}, \mathfrak{y}\right|+\left|B_{1}^{* 1} \mathfrak{x}_{1}+B_{1}^{* 2} \mathfrak{x}_{2}, \mathfrak{x}_{2}, \mathfrak{y}\right|\right\} \\
& +\left|B_{1}^{* 1} \mathfrak{\Upsilon}_{1}+B_{1}^{* 2} \mathfrak{x}_{2}, B_{2}^{* 1} \mathfrak{x}_{1}+B_{2}^{* 2} \mathfrak{\swarrow}_{2}, \mathfrak{y}\right|=0,
\end{aligned}
$$

consequently

$$
\frac{1}{R^{2}}+\frac{1}{R}\left(B_{1}^{* 1}+B_{2}^{* 2}\right)+\left(B_{1}^{* 1} B_{2}^{* 2}-B_{1}^{* 2} B_{2}^{* 1}\right)=0 .
$$

Denoting the roots of this equation by $\frac{1}{R_{1}}$ and $\frac{1}{R_{2}}$, we obtain

$$
H \equiv \frac{1}{2}\left(\frac{1}{R_{1}}+\frac{1}{R_{2}}\right)=-\frac{B_{1}^{* 1}+B_{2}^{* 2}}{2}=-\frac{B_{i}^{* i}}{2},
$$

and

$$
K \equiv \frac{1}{R_{1} R_{2}}=B_{1}^{* 1} B_{2}^{* 2}-B_{1}^{* 2} B_{2}^{* 1}
$$

1/R are called the r-affine principal curvatures and $R_{i}$ are called the radii of the r-affine principal curvatures.

The point

$$
\jmath_{i}=\mathfrak{x}+R_{i} \mathfrak{y} \quad(i=1,2)
$$

are called the centres of the r-affine principal curvatures. 
Eliminating $R$ and $d R-\sigma$ from (40), then we have

$$
\left|\mathfrak{r}_{i} d u^{i}, B_{k}^{\alpha} r_{\alpha} d u^{k}, \mathfrak{y}\right|=0 \text {, }
$$

then

$$
\left|\mathfrak{x}_{i}, B_{k}^{\alpha} \mathfrak{r}_{\alpha}, \mathfrak{y}\right| d u^{i} d u^{k}=0,
$$

therefore

$$
B_{1}^{2} d u^{1} d u^{2}+\left(B_{2}^{2}-B_{1}^{1}\right) d u^{1} d u^{2}-B_{2}^{1} d u^{2} d u^{1}=0 .
$$

The equation (46) gives the parametric equation of the $r$-affine lines of curvature.

Thus we have the following.

Theorem 1. A necessary and sufficient condition that the r-affine lines of curvature be parametric is that the conditions.

$$
B_{1}^{2}=B_{2}^{1}=0, \quad B_{2}^{2}=B_{1}^{1}
$$

hold, and $R_{i}$ are defined as

$$
R_{i}=-\frac{1}{B_{i}^{i}} .
$$

Denote the affine perpendicular distance from the centre $z_{i}$ of the $r$-affine normal curvature to $x$ by $\mathfrak{p}_{i}$ and denote the $r$-affine perpendicular distance of $x$ from $z_{i}$ by $\mathfrak{r}_{i}$, then we have

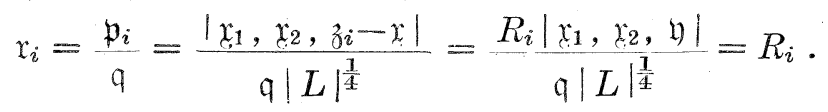

This gives a geometrical meaning of $R_{i}$

\section{$\S 4$. Conditions of integrability.}

From (10) and (24) we have

$$
\begin{aligned}
\mathfrak{x}_{i k} & \left.=A_{i k}^{\alpha} \mathfrak{x}_{\alpha}+\frac{\mathfrak{q}_{i}}{\mathfrak{q}} \mathfrak{r}_{k}+\frac{\mathfrak{q}_{k}}{\mathfrak{q}} \mathfrak{r}_{i}+\frac{g_{i k}}{\mathfrak{q}} \mathfrak{l}\right) \\
& =\left(A_{i k}^{\alpha}+\frac{\mathfrak{q}_{i}}{\mathfrak{q}} \delta_{k}^{\alpha}+\frac{\mathfrak{q}_{k}}{\mathfrak{q}} \delta_{i}^{\alpha}\right) \mathfrak{x}_{\alpha}+\frac{g_{i k}}{\mathfrak{q}} \mathfrak{y},
\end{aligned}
$$

putting

$$
T_{i k}^{\alpha}=A_{i k}^{\alpha}+\frac{\mathfrak{q}_{i}}{\mathfrak{q}} \delta_{k}^{\alpha}+\frac{\mathfrak{q}_{k}}{\mathfrak{q}} \delta_{i}^{\alpha},
$$


or

$\left(50^{\prime}\right)$

$$
T_{i k, l}^{\alpha}=A_{i k, l}+\frac{\mathfrak{q}_{i}}{\mathfrak{q}} g_{k l}+\frac{\mathfrak{q}_{k}}{\mathfrak{q}} g_{i l},
$$

we obtain

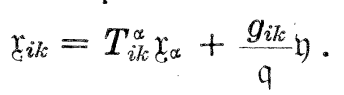

By differentiating (51) covariantly with respect to $u^{l}$ we have

$$
\begin{aligned}
\mathfrak{x}_{i k, l} & =T_{i k \mathfrak{\alpha} \alpha l}^{\alpha}+T_{i k, l}^{\alpha} \mathfrak{k}_{\alpha}+\frac{\mathfrak{q}_{i k}}{\mathfrak{q}} \mathfrak{y}_{l}-\frac{g_{i k} \mathfrak{q}_{l}}{\mathfrak{q}^{2}} \mathfrak{y} \\
& =T_{i k}^{\alpha}\left(T_{\alpha l}^{\beta} \mathfrak{x}_{\beta}+\frac{g_{\alpha l}}{\mathfrak{q}} \mathfrak{y}\right)+T_{i k, l}^{\alpha} \mathfrak{k}_{\alpha}+\frac{g_{i k}}{\mathfrak{q}} \mathfrak{q} B_{l}^{\alpha} \mathfrak{x}_{\alpha}-\frac{g_{i k} \mathfrak{q}_{l}}{\mathfrak{q}^{2}} \mathfrak{y},
\end{aligned}
$$

then

$$
\mathfrak{x}_{i k, l}=\left(T_{i k}^{\sigma} T_{\sigma l}^{\alpha}+T_{i k, l}^{\alpha}+g_{i k} B_{l}^{\alpha}\right) \mathfrak{x}_{\alpha}+\frac{1}{\mathfrak{q}^{2}}\left(\mathfrak{q} T_{i k}^{\alpha} g_{\alpha l}-g_{i k} \mathfrak{q}_{l}\right) \mathfrak{y} .
$$

From the equation

$$
\mathfrak{x}_{i k, l}=\mathfrak{x}_{i l, k}
$$

we have

$$
T_{i k}^{\sigma} T_{\sigma l}^{\alpha}+T_{i k, l}^{\alpha}+g_{i k} B_{l}^{\alpha}=T_{i l}^{o} T_{\sigma k}^{\alpha}+T_{i l, k}^{\alpha}+g_{i l} B_{k}^{\alpha}
$$

and

$$
\mathrm{q} T_{i k, l}-g_{i k} \mathfrak{q}_{l}=\mathfrak{q} T_{i l, k}-g_{i l} \mathfrak{q}_{k} .
$$

By means of $\left(50^{\prime}\right)$ the relation $(55)$ becomes

$$
\mathfrak{q} A_{i k, l}-2 g_{i k} \mathfrak{q}_{l}=\mathfrak{q} A_{i l, k}-2 g_{i l} \mathfrak{q}_{k} .
$$

By multiplying (54) by $g^{i k}$, we have

$$
B_{l}^{\alpha}=g^{i k}\left(T_{i l}^{\sigma} T_{\sigma k}^{\alpha}+T_{i l, k}^{\alpha}-T_{i k}^{\alpha} T_{\sigma l}^{\alpha}-T_{i k, l}^{\alpha}\right)
$$

As a consequence from the conditions of apolarity

$$
g^{i k} A_{i k, l}=0 \text {, }
$$


and

$$
g^{i k, m}=0
$$

we obtain

$$
g^{i k} A_{i k, l m}=0
$$

then

$$
g^{i k} T_{i k, l}=\frac{2 \mathfrak{q}_{l}}{\mathfrak{q}}
$$

and

$$
g^{i k} T_{i k, l m}=2\left(\frac{\mathfrak{q}_{l}}{\mathfrak{q}}\right)_{m}
$$

By means of (59) and (60) the relation (57) becomes

$$
B_{l m}=g^{i k} T_{i l}^{\sigma} T_{o k, m}+g^{i k} T_{i l . m k}-\frac{2 \mathfrak{q}_{n}}{\mathfrak{q}} g^{n \sigma} T_{\sigma l, m}-2\left(\frac{\mathfrak{q}_{m}}{\mathfrak{q}}\right)_{l}
$$

By covariant differentiation of (27) with respect to $u^{k}$ we have

$$
\begin{aligned}
\mathfrak{y}_{i k^{\prime}} & =\mathfrak{q} B_{i}^{\alpha} \mathfrak{x}_{\alpha k_{k}}+\left(\mathfrak{q} B_{i}^{\alpha}\right)_{k} \mathfrak{x}_{\alpha} \\
& =B_{i}^{\alpha}\left(\xi_{\alpha k}+\mathfrak{q}_{\alpha} \mathfrak{x}_{k}+\mathfrak{q}_{k} \mathfrak{x}_{\alpha}\right)+\left(\mathfrak{q} B_{i}^{\alpha}\right)_{k} \mathfrak{x}_{\alpha} \\
& =B_{i}^{\alpha}\left(\mathfrak{q} A_{\alpha k k}^{\beta} \mathfrak{x}_{\beta}+g_{\alpha k} \mathfrak{y}+\mathfrak{q}_{\alpha} \mathfrak{x}_{k}+\mathfrak{q}_{k} \mathfrak{x}_{\alpha}\right)+\left(\mathfrak{q} B_{i}^{\alpha}\right)_{k} \mathfrak{x}_{\alpha} \\
& =\left\{\mathfrak{q} B_{i}^{\alpha} A_{\alpha k}^{\beta}+\mathfrak{q}_{\alpha} B_{i}^{\alpha} \delta_{k}^{\beta}+\mathfrak{q}_{k} B_{i}^{\beta}+\left(\mathfrak{q} B_{i}^{\beta}\right)_{k}\right\} \mathfrak{x}_{\beta}+B_{i k} \mathfrak{y},
\end{aligned}
$$

Then as the condition of integrability of (27) we obtain

$$
\begin{aligned}
& \mathfrak{q} B_{i}^{\alpha} A_{\alpha k}^{\beta}+\mathfrak{q}_{\alpha} B_{i}^{\alpha} \delta_{k}^{\beta}+2 \mathfrak{q}_{k} B_{i}^{\beta}+\mathfrak{q} B_{i, k}^{\beta} \\
= & \mathfrak{q} B_{k}^{\alpha} A_{\alpha i}^{\beta}+\mathfrak{q}_{\alpha} B_{k}^{\alpha} \delta_{i}^{\beta}+2 \mathfrak{q}_{i} B_{k}^{\beta}+\mathfrak{q} B_{k, i}^{\beta}
\end{aligned}
$$

or

$$
\begin{aligned}
& \mathfrak{q} B_{i}^{\alpha} A_{\alpha k, l}+\mathfrak{q}_{\alpha} B_{i}^{\alpha} g_{k l}+2 \mathfrak{q}_{k} B_{i l}+\mathfrak{q} B_{i, l k} \\
= & \mathfrak{q} B_{k}^{\alpha} A_{\alpha i, l}+\mathfrak{q}_{\alpha} B_{l}^{\alpha} g_{i l}+2 \mathfrak{q}_{i} B_{k l}+\mathfrak{q} B_{k, l i} .
\end{aligned}
$$

We can find that the equation (38) is integrable by virtue of the conditions (56), (57) (or (61)) and (63) (or $\left(63^{\prime}\right)$ ). Thus the conditions of in- 
tegrability for the fundamental equations (24), (27) and (38) are given by the equations (56), (57) (or (61)) and (63) (or $\left(63^{\prime}\right)$ ). Hence as in the euclidean space $\left({ }^{20}\right)$ we have the fundamental

Theorem 2. When $g_{i k}$ and $A_{i k}, l$ are given as the functions of parameters of $u^{1}$ and $u^{2}$ of the unit surface, satisfying equatins (22), (56), (57) (or (61)) and (63) (or $\left.\left(63^{\prime}\right)\right)$ and these functions are differentiable up to the second order, there exist a surface for which these functions are the coefficients of the fundamental forms $g_{i k} d u^{i} d u^{k}$ and $A_{i k}{ }_{l} d u^{i} d u^{k} d u^{l}$, except for the translation with respect to the unit surface defined by a given function $E(u, v)$, unless the unit surface is a periodic surface or a surface of revolution or a quadric, and if the unit surface is a periodic surface or a surface of revolution or a quadric, there exists one and only one surface, except for the equivalent affine transformation.

\section{R-Affine sphere.}

When the $r$-affine normals of a surface $F$ pass through a fixed point $z$, the surface $F$ is called the r-affine sphere. If the surface is a $r$-affine sphere, its $r$-affine normals envelope a fixed point $z$. Then from (44) we have

$$
\mathfrak{z}=\mathfrak{y}+R_{i} \mathfrak{y},
$$

where $R_{1}=R_{2}=$ constant $=R(\neq \pm \infty)$. Consequentlv we obtain

$$
H=\frac{1}{R}=\text { constant, } K=\frac{1}{R^{2}}=\text { constant } \text {. }
$$

Take the point $z$ as the origin $O$, then from (6) and (48) we have

$$
\mathfrak{r}=\frac{\mathfrak{p}}{\mathfrak{q}}=\frac{\left|x_{1}, x_{2},-\mathfrak{x}\right|}{\left|\mathfrak{e}_{1}, \mathfrak{e}_{2},-\mathfrak{e}\right|}\left|K^{*}\right|^{\frac{3}{4}}=R=\text { constant }
$$

Substituting (1)

$$
\begin{aligned}
\mathfrak{r}=\frac{\mathfrak{y}}{q}=\frac{\left|x_{1}, x_{2},-\mathfrak{x}\right|}{\left|a_{1}^{\sigma} \tilde{x}_{\sigma}, a_{2}^{*} x_{\tau},-e\right|}\left|K^{*}\right|^{\frac{3}{4}} & =\frac{\left|x_{1}, x_{2},-x\right|}{a\left|x_{1}, x_{2},-e\right|}\left|K^{*}\right|^{\frac{3}{4}} \\
& =\frac{p\left|K^{*}\right|^{\frac{3}{4}}}{q}=R,
\end{aligned}
$$

(20) Loc. cit. 
where $p$ and $q$ denote respectively the perpendicular distance from $O$ to the corresponding tangent planes.

In case where $K^{*}>0$, from (3) we have

$$
r=\frac{p}{q}=R K^{* \frac{7}{3}}=R\left(\frac{D}{g}\right)^{\frac{1}{4}},
$$

and by a symmetric transformation

$$
\hat{x}=R^{2} \bar{r}
$$

we can make so that

$$
\mathfrak{p}=\mathfrak{q} \quad \text { or } \quad r=\frac{p}{q}=K^{* \frac{1}{4}}=\left(\frac{\bar{K}(\mathfrak{x})}{\bar{K}(\mathfrak{e})}\right)^{\frac{1}{4}}=\frac{P}{Q},
$$

where $P$ and $Q$ denote respectively the perpendicular distances from the origin to the tangent planes at the corresponding points on the indicatrices of their affine curvatures. $\left({ }^{21}\right)$

Let us put

$$
\frac{p}{P}=\frac{q}{Q}=\lambda,
$$

then we have

$$
(-\mathrm{r} \xi)=\lambda(\overline{\mathrm{y}} \xi),
$$

where $\xi$ denotes the surface normal vector and $\bar{y}$ denotes the affine normal vector at $\mathrm{x}$.

Consequently we can put

$$
x+2 \bar{y}=b^{\sigma} x_{\sigma},
$$

then by cavariant differentiation with respect to $u^{i}$ and by means of the elementary fundamental equations we have

$$
\mathfrak{x}_{i}+\lambda \bar{B}_{i}^{\alpha} \mathfrak{x}_{\alpha}+\lambda_{i} \overline{\mathfrak{y}}=b^{\sigma}\left(\bar{A}_{\sigma i}^{\alpha} \mathfrak{x}_{\sigma}+g_{\sigma i} \overline{\mathfrak{y}}\right)+b_{i}^{\sigma} \mathfrak{c}_{\sigma},
$$

then

$$
b^{\sigma} g_{\sigma i}=\lambda_{i},
$$

(21) The relation $P=|K(x)|^{\frac{1}{4}}$ is easily seen from the equation (186) in 864 , W. Blaschke, Diff. Geom. II, p. 166. 
and

(75)

$$
\begin{aligned}
1+\lambda \bar{B}_{1}^{1} & =b^{\sigma} \bar{A}_{\sigma 1}^{1}+b, \frac{1}{1}, \\
\lambda \bar{B}_{1}^{2} & =b^{\sigma} \bar{A}_{\sigma 1}^{2}+b_{, 1}^{2}, \\
1+\lambda \bar{B}_{2}^{2} & =b^{\sigma} \bar{A}_{\sigma 2}^{2}+b, 2, \\
\lambda \bar{B}_{2}^{1} & =b^{\sigma} \bar{A}_{o 2}^{1}+b, 2,
\end{aligned}
$$

From (74) we have

$$
b^{i}=\lambda_{\alpha} g^{\alpha i}
$$

By means of (76) and $\bar{y}=\frac{1}{2} g^{\alpha \beta} \mathfrak{x}_{\alpha \beta}$ the equation (73) becomes

$$
x+\lambda y-\lambda_{\alpha} x_{\beta} g^{\alpha_{\beta}}=0 .
$$

From (1) we have

$$
\mathfrak{x}_{\alpha}=-\bar{a}_{\alpha}^{i} \mathfrak{e}_{i}
$$

where $\bar{a}_{i}^{j}=\left(\right.$ cofactor of $\left.a_{i}^{j}\right) / a=-\frac{1}{\bar{a}} L_{i \alpha} l^{\alpha j}$.

By differentiating covariantly (1') with respect to $u^{\beta}$ we obtain

$$
\mathfrak{x}_{\alpha \beta}=-\bar{a}_{\alpha}^{i} \mathfrak{e}_{i, \beta}-\bar{a}_{\alpha, \beta}^{i} \mathfrak{e}_{i},
$$

and substituting $\left(1^{\prime}\right)$ and $(78)$ in $\overline{\mathfrak{y}}$ and $\overline{\mathfrak{y}}_{\mathrm{r}}$

$$
\begin{gathered}
\overline{\mathfrak{y}}=-\frac{1}{2} g^{\alpha \beta}\left(\bar{a}_{\alpha}^{i} \mathfrak{e}_{i, \beta}+\bar{a}_{\alpha, \beta}^{i} \mathfrak{e}_{i}\right), \\
\overline{\mathfrak{y}}_{\curlyvee}=\bar{B}_{\curlyvee}^{j} \hat{x}_{j}=-\bar{B}_{\curlyvee}^{j} \bar{a}_{j}^{i} \mathfrak{e}_{i} .
\end{gathered}
$$

Differentiate covariantlv (77) with respect to $u^{r}$, and substitute $\left(1^{\prime}\right)$, (79) and (80), then we have

$$
\begin{aligned}
\left(\bar{a}_{\curlyvee}^{i}+\lambda \bar{B}_{\curlyvee}^{j} \bar{a}_{j}^{i}\right. & \left.+\frac{1}{2} \lambda_{\curlyvee} g^{\alpha_{\beta}} \bar{a}_{\alpha, \beta}^{i}-\lambda_{\alpha \curlyvee} g^{\alpha_{\beta}} \bar{a}_{\beta}^{i}-\lambda_{\alpha} g^{\alpha_{\beta}} \bar{a}_{\beta, \gamma}^{i}\right) e_{i} \\
+ & \frac{1}{2} \lambda_{\curlyvee} g^{\tau \mu} \bar{a}_{\tau}^{i} e_{i, \mu}-\lambda_{\alpha} g^{\alpha_{\beta}} \bar{a}_{\beta}^{i} \mathfrak{e}_{i, \gamma}=0
\end{aligned}
$$

From (71) in the same manner as we have obtained (77) we have the equation 


$$
e+\lambda \bar{\eta}-\lambda_{\alpha} \bar{g}^{\alpha_{\beta}} e_{3}=0
$$

By differentiating covariantly (82) with respect to $u^{i}$ we have

$$
\frac{1}{2} \lambda_{i} \bar{g}^{\tau \mu} \mathfrak{e}_{\tau \mu}-\lambda_{\alpha} \bar{g}^{\alpha \beta} \mathfrak{e}_{\beta i}=-\mathfrak{e}_{i}-\lambda b_{i}^{\sigma} \mathfrak{e}_{\sigma}+\lambda_{\alpha i} \bar{g}^{\alpha \sigma} \mathfrak{e}_{\sigma},
$$

where $b_{i}^{k}$ denote the corresponding quantities on $E(\mathfrak{e})$ to $\bar{B}_{i}^{k}$ on $F(\mathfrak{c})$. The relation between $e_{i, k}$ and $e_{i k}$ is given by the equation

$$
\mathfrak{e}_{i, k}=\mathfrak{e}_{i k}+\left(\bar{\Gamma}_{i k}^{\sigma}-\Gamma_{i k}^{\sigma}\right) \mathfrak{e}_{\sigma},
$$

where $\bar{\Gamma}_{i k}^{\sigma}$ and $\Gamma_{i k}^{\sigma}$ denote respectively Christoffel's symbol concerning respectively $\bar{g}_{i k}$ and $g_{i k}$.

Substituting (83) and (84) in (81) we have

$$
\begin{array}{r}
g_{k l}-\bar{g}_{k l}+\lambda\left(\bar{B}_{k l}-b_{k l}\right)+\frac{1}{2} \lambda_{k}\left(\frac{\left|K^{*}\right| . l}{\left|K^{*}\right|}+\Gamma_{\mu l}^{\mu}-\bar{\Gamma}_{\mu l}^{\mu}\right) \\
+\frac{1}{4} \lambda_{l} \frac{\left|K^{*}\right| \cdot k}{\left|K^{*}\right|}+2 \lambda_{\alpha}\left(\Gamma_{k l}^{\alpha}-\bar{\Gamma}_{k l}^{\alpha}\right)=0 .
\end{array}
$$

Hence we have the following

Theorem 3. If the fundamental tensor $g_{i j}$ satisfy the equation (85) and the provided fundamental equations, and the radius vector $\mathfrak{x}$ of the point on a curve is defined as a solution of the differential equation (7n), the curve is necessarily an unit $r$-affine sphere, and its converse is also true. The r-affine sphere is a symmetric transformation of an unit r-affine sphere.

\section{$\$ 6$. Indicatrix of r-principal curvatures.}

The indicatrix of r-principal curvatures is defined as the surface described the end points of the $r$-affine normal vector $-\mathfrak{y}$ from the origin.

From (27) we have the following

Theorem 4. The tangent planes at $\mathfrak{x}$ and $\mathfrak{y}$ are parallel to each other.

Denote the surface normal by $\xi$, then we have

$$
(\xi \mathfrak{y})=\mathfrak{q}|\bar{K}|^{\mathfrak{x}}
$$

According to the correspondence of the parallel tangents by means of (1) and (3) we have 


$$
K=B_{1}^{1} B_{2}^{2}-B_{1}^{2} B_{2}^{1}=\frac{\bar{K}(\mathfrak{y})}{\bar{K}(\mathfrak{y})} .
$$

Hence a geometrical meaning of the $r$-affine total curvature is obtained as follows:

Theorem 5. The r-affine total curvuture means the ratio of the elementary total curvatures at a point $\mathfrak{x}$, on a surface $F(\mathfrak{x})$, and a point $\mathfrak{y}$, on its indicatrix of r-a.ffine curvatures.

Differentiating (27) covariantly with respect to $u^{k}$ we have

$$
\mathfrak{y}_{i k}=B_{i}^{* \sigma} \mathfrak{x}_{\sigma k}+B_{i . k}^{* \sigma} \mathfrak{x}_{\sigma},
$$

then putting

consequently

$$
\begin{aligned}
L_{0, i k} & =\left|-\mathfrak{y}_{i k},-\mathfrak{y}_{1},-\mathfrak{y}_{2}\right| \\
& =-\left|B_{i}^{* \sigma} \hat{\gamma}_{\sigma k}, B_{1}^{* \tau} \mathfrak{x}_{\tau}, B_{2}^{* \mu} \mathfrak{x}_{\mu}\right| \\
& =-K B_{i}^{* \sigma} L_{\sigma k},
\end{aligned}
$$

$$
L_{0}=L_{0,11} L_{0,22}-L_{0,12}^{2}=K^{3} L .
$$

Let $\mathfrak{r}_{0}$ denote the $r$-perpendicular distance from the origin to $\mathfrak{y}$, then we have

$$
\mathfrak{r}_{0}=\frac{\left|\mathfrak{y}, \mathfrak{y}_{u}, \mathfrak{y}_{v}\right|}{\mathfrak{q}\left|L_{0}\right|^{\frac{1}{4}}}=\frac{\left|\mathfrak{y}, B_{1}^{* \lambda} \mathfrak{x}_{\lambda}, B_{1}^{* \mu} \mathfrak{f}_{\mu \mu}\right|}{\mathfrak{q}|K|^{\frac{3}{4}}|L|^{\frac{1}{4}}}=\frac{K}{|K|^{\frac{3}{4}}}= \pm|K|^{\frac{1}{4}} .
$$

Thus we get the following

Theorem 6. A necessary and sufficient condition that the r-affine total curvature be constant is that the indicatrix of r-affine curvatures is a r-affine sphere.

Let $d O(\mathfrak{x})$ and $d O(\mathfrak{y})$ denote respectively the affine areal elements of the surface $F(\mathfrak{x})$ and its indicatrix of $r$-affine curvatures, then from (90) we have

$$
\frac{d O(\mathfrak{y})}{d O(\mathfrak{x})}=|K|^{\frac{3}{4}}
$$

and

$$
O(\mathfrak{y})=\int|K|^{\frac{3}{4}} d O(\mathfrak{x}) .
$$

Assume that the indicatrix of $r$-affine curvatures is a convex closed surface and denote its volume by $V_{0}$, then we have 


$$
V_{0}=\frac{1}{3} \iint\left|-\mathfrak{y},-\mathfrak{y}_{u},-\mathfrak{y} v\right| d u d v=\frac{1}{3} \iint(-\mathfrak{q}) K|L|^{\frac{1}{4}} d u d v .
$$

Let $\Omega$ denote the relative surface area (=Relativobegfläche) $\left({ }^{22}\right)$ between the unit surface and the indicatrix of $r$-affine curvature, then we have

$$
d \Omega=\frac{1}{3}\left|\mathfrak{e}, \mathfrak{y}_{u}, \mathfrak{y}_{v}\right| d u d v=\frac{1}{3}\left|\mathfrak{e}, \mathfrak{x}_{u}, \mathfrak{x}_{v}\right| K d u d v,
$$

substituting (1)

$$
\begin{aligned}
d \Omega & =\frac{1}{3}\left|\mathfrak{e},-\frac{1}{a}\left(a_{2}^{2} \mathfrak{e}_{1}-a_{1}^{2} \mathfrak{e}_{2}\right),-\frac{1}{a}\left(a_{1}^{1} \mathfrak{e}_{2}-a_{2}^{1} \mathfrak{e}_{1}\right)\right| K d u d v \\
& =\frac{1}{3}\left|\mathfrak{e}, \mathfrak{e}_{1}, \mathfrak{e}_{2}\right| \frac{K}{a} d u d v \\
& =\frac{1}{3}(-\mathfrak{q})|l|^{\frac{1}{4}} \frac{K}{K^{*}} d u d v .
\end{aligned}
$$

By means of (94) and (95) we obtain

$$
V_{0}=\int K^{*}\left|\frac{L}{l}\right|^{\frac{1}{4}} d \Omega=\int K^{* \frac{1}{4}} d \Omega .
$$

Using Cauchy's inequality, we have the following

$$
V_{0}^{4}=\left(\int K^{* \frac{1}{4}} d \Omega\right)^{4} \leqq\left(\int K^{* \frac{1}{2}} d \Omega\right)\left(\int d \Omega\right)^{2} \leqq\left(\int K^{*} d \Omega\right)\left(\int d \Omega\right)^{3}
$$

then

$$
V_{0}^{4} \leqq \Omega^{3} \int K^{*} d \Omega=\Omega^{3} \int K \frac{\bar{K}(\mathfrak{y})}{\bar{K}(\mathfrak{e})} d \Omega=\Omega^{3} \int K d V(\mathfrak{e})
$$

\section{$\S 7$. Surfaces of constant $r$-affine breadth.}

We will make here an extension of the affine breadth $\left(^{23}\right)$ as in $\S 7$, chapter I: Take a convex closed surface $F(\mathfrak{x})$ and denote the points $P_{1}$ and $P_{2}$, such that the tangent planes at $P_{1}$ and $P_{2}$ are parallel to each other by $\mathfrak{x}_{1}$ and $\mathfrak{x}_{2}$. And denote the surface normal and the $r$-affine surface normal at $P_{i}$ by $\xi_{i}$ and $\mathfrak{y}_{i}$, then the $r$-affine brerdth $\mathfrak{b}$ is defined as follows

(22) H. Minikowski, Volumen und Oberflächen, Math, Ann., 57 (1903).

(23) J. Hirakawa, The affine breadth and its relation to the relative breadth, Jap. Journ. of Math., XII (1935). 


$$
\mathfrak{b}=2 \frac{\left(\mathfrak{x}_{2}-\mathfrak{x}_{1}\right) \xi_{1}}{\left(\mathfrak{y}_{1} \xi_{1}\right)+\left(\mathfrak{h}_{2} \xi_{2}\right)}
$$

However by the definition of the $r$-affine normal we have

$$
\begin{aligned}
\mathfrak{y} \xi & =\frac{1}{2} g^{\alpha_{\beta}} \xi_{\alpha \beta} \xi=\frac{1}{2} g^{\alpha_{\beta}}\left(\mathfrak{q} \mathfrak{x}_{\alpha \beta}-\mathfrak{q}_{\alpha} \mathfrak{k}_{\beta}-\mathfrak{q}_{\beta} \mathfrak{x}_{\alpha}\right) \xi \\
& =\mathfrak{q}(\overline{\mathfrak{y}} \xi) \\
& =\mathfrak{q}|\bar{K}|^{\frac{1}{4}},
\end{aligned}
$$

where $\bar{y}$ denotes the elementary affine surface normal.

Let us denote the function of supporting lines at $\mathfrak{x}_{i}$ by $p_{i}$, then the $r$-affine breadth is also given by the following expression

$$
\mathfrak{b}=\frac{2\left(p_{1}+p_{2}\right)}{\mathfrak{q}_{1} \bar{K}_{1}^{\frac{1}{4}}+\mathfrak{q}_{2} \bar{K}_{2}^{\frac{1}{4}}}
$$

Let $\mathfrak{p}_{i}$ and $t_{i}$ denote the affine perpendicular distance and the relative one from $x_{i}$ to $x_{k}(i, k=1,2 ; i \neq k)$, then the following relation, is obtained:

$$
\frac{2}{b}=\frac{1}{\frac{\left(x_{2}-\mathfrak{x}_{1}\right) \xi_{1}}{\mathfrak{q}_{1} \bar{K}_{1}^{\frac{1}{4}}}}+\frac{1}{\frac{\left(\mathfrak{x}_{1}-\mathfrak{x}_{2}\right) \xi_{2}}{\mathfrak{q}_{2} \bar{K}_{2}^{\frac{1}{4}}}}=\frac{1}{\frac{p_{1}}{q_{1}}}+\frac{1}{\frac{p_{2}}{q_{2}}}=\frac{1}{t_{1}}+\frac{1}{t_{2}} .
$$

Hence we have the following

Theorem 7. The r-affine breadth $\mathfrak{b}$ is equal to the harmonic mean of the $r$-affine perpendicular distance $t_{1}$ and $t_{2}$, and then $\mathfrak{b}$ is an affine invariant.

Theorem 8. A necessary and sufficient condition that the affine breadth of an ovaloid $E$ be constant, is that the two vectors $\mathfrak{x}_{1}-\mathfrak{x}_{2}$ and $\mathfrak{y}_{1}-\mathfrak{y}_{2}$ are parallel.

The proof is given by means of the fundamental equation (27).

Theorem 9. If the affine normals at the points of contact of the parallel tangent planes on an ovaloid are a r-affine double normal, this surface has the following properties:

$1^{\circ}$. The ovaloid is a surface of constant r-affine breadth.

2 . The indicatrix of r-affine curvature is central. 
$3^{\circ}$. The r-affine perpendicular distance between two points $x_{1}$ and $x_{2}$ is constant $(=\mathfrak{b})$.

$4^{\circ}$. The sum of the two radii $R_{1},{ }_{i}$ and $R_{2},{ }_{i}$ of $r$-affine principal curvatures is constant $(=\mathfrak{b})$.

$5^{\circ}$. The sum of the r-affine perpendicular distances $\mathfrak{x}_{1}$ and $\mathfrak{x}_{2}$ from the origin to $\mathfrak{x}_{1}$ and $\mathfrak{r}_{2}$ is constant $(=\mathfrak{b})$.

Proof. By the assumption and Theorem 8 we have

$$
\mathfrak{b}=\text { constant }
$$

and

$$
\mathfrak{x}_{1}-\mathfrak{x}_{2}+\frac{\mathfrak{b}}{2}\left(\mathfrak{y}_{1}-\mathfrak{y}_{2}\right)=0 \text {. }
$$

From the assumption we can put

$$
\mathfrak{y}_{1}=-\sigma \mathfrak{y}_{2} .
$$

By differentiation we find that $\sigma=$ constant $=1$, so that we have

$$
\mathfrak{y}_{1}+\mathfrak{y}_{2}=0
$$

Therefore the indicatrix of $r$-principal curvatures is central. Now we have

$$
\mathrm{t}_{1}=\frac{\left(\mathfrak{x}_{2}-\mathfrak{x}_{1}\right) \xi_{1}}{\mathfrak{q}_{1} \bar{K}_{1}^{\bar{t}^{4}}}=\frac{\left(\mathfrak{x}_{1}-\mathfrak{x}_{2}\right) \xi_{2}}{\mathfrak{q}_{2} \bar{K}_{2}^{\frac{1}{4}}}=\mathrm{t}_{2}=\mathfrak{b}=\text { constant }
$$

By means of (102) and (103) we obtain

$$
\mathfrak{x}_{2}=\mathfrak{x}_{1}+\mathfrak{b} \mathfrak{y}_{1}, \quad \mathfrak{x}_{1}=\mathfrak{x}_{2}+\mathfrak{b} \mathfrak{y}_{2}
$$

By differentiation of (105) with respect to respectively $u$ and $v$, and using (27) the following equations are obtained:

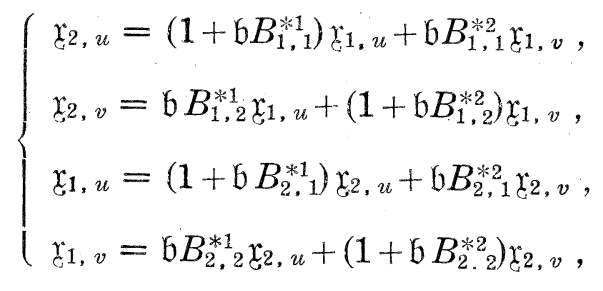


Take the $r$-affine lines of curvature as parametric curves, then (106) becomes

$$
\left\{\begin{array}{l}
\mathfrak{x}_{2, u}=\left(1+\mathfrak{b} B_{1,1}^{* 1}\right) \mathfrak{x}_{1, u}, \\
\mathfrak{x}_{2, u}=\left(1+\mathfrak{b} B_{1,2}^{* 2}\right) \mathfrak{x}_{1}, v, \\
\mathfrak{x}_{1, u}=\left(1+\mathfrak{b} B_{2,1}^{* 1}\right) \mathfrak{x}_{2, u}, \\
\mathfrak{x}_{1, v}=\left(1+\mathfrak{b} B_{2,2}^{* 2}\right) \mathfrak{c}_{2, v},
\end{array}\right.
$$

Hence by Theorem 1. we have

$$
\mathfrak{b}=-\left(\frac{1}{B_{1.1}^{* 1}}+\frac{1}{B_{2,1}^{* 11}}\right)=R_{1,1}+R_{2,1},
$$

$$
\mathfrak{b}=-\left(\frac{1}{B_{1,2}^{* 2}}+\frac{1}{B_{2,2}^{* 2}}\right)=R_{1,2}+R_{2,2}
$$

Let $\mathfrak{r}_{i}$ denote the $r$-affine perpendicular distance from the origin to $x_{i}$, then we have

$$
\mathfrak{x}_{1}=\frac{\left(-\mathfrak{x}_{1}, \xi_{1}\right)}{\left(\mathfrak{y}_{1} \xi_{1}\right)}=\frac{-\left(-\mathfrak{x}_{2}-\mathfrak{b} \mathfrak{y}_{2}\right) \xi_{2}}{\left(\mathfrak{y}_{2} \xi_{2}\right)}=-\frac{\left(-\mathfrak{x}_{2}\right) \xi_{2}}{\left(\mathfrak{y}_{2} \xi_{2}\right)}+\mathfrak{b} \frac{\left(\mathfrak{y}_{2} \xi_{2}\right)}{\left(\mathfrak{y}_{2} \xi_{2}\right)}=-\mathfrak{x}_{2}+\mathfrak{b},
$$

therefore

$$
\mathfrak{x}_{1}+\mathfrak{r}_{2}=\mathfrak{b}=\text { constant }
$$

Tôkyô Butsuri-Gakkô. 